\title{
Numerical Investigation of Sound Generation due to Laminar Flow Past Elliptic Cylinders
}

\author{
Bikash Mahato, Naveen Ganta and Yogesh G. Bhumkar* \\ Scientific Computing Laboratory, School of Mechanical Sciences, Indian \\ Institute of Technology Bhubaneswar, Odisha, India
}

Received 13 March 2019; Accepted (in revised version) 5 September 2019

\begin{abstract}
Numerical investigation of sound generation due to unsteady laminar flow past elliptic cylinders has been carried out using direct numerical simulation $(D N S)$ approach at a free-stream Mach number of 0.2. Effects of aspect ratio $(0.6 \leq A R \leq 1.0)$ and Reynolds number $(100 \leq R e \leq 160)$ on the characteristics of radiated sound fields are analyzed. Two-dimensional compressible fluid flow equations are solved on a refined grid using high resolution dispersion relation preserving $(D R P)$ schemes. Using present $D N S$ data, equivalent noise sources as given by various acoustic analogies are evaluated. Amplitudes and frequencies associated with these noise sources are further related to characteristics of disturbance pressure fields. Disturbance pressure fields are intensified with increase in Reynolds number and aspect ratio. Thus, radiated sound power increases with increase in Reynolds number and aspect ratio. Among various cases studied here, minimum and maximum values of radiated sound power are found at $R e=120 \& A R=0.6$ and $R e=160 \& A R=1.0$, respectively. Directivity patterns show that the generated sound fields are dominated by the lift dipole for all cases. Next, proper orthogonal decomposition $(P O D)$ technique has been implemented for decomposing disturbance pressure fields. The $P O D$ modes associated with the lift and the drag dipoles have been identified. $P O D$ analyses also clearly display that the radiated sound fields are dominated by the lift dipole only. Further, acoustic and hydrodynamic modes obtained using Doak's decomposition method have confirmed the patterns of radiated sound field intensities.
\end{abstract}

AMS subject classifications: 76Q05, 76M20, 76G25

Key words: Computational aeroacoustics, acoustic analogies, aeolian tones, proper orthogonal decomposition, Doak's decomposition.

\section{Introduction}

Historically, researchers have shown immense interest in analyzing external flows past streamlined and bluff bodies. Some of the important applications can be listed

${ }^{*}$ Corresponding author. Email addresses: bm18@iitbbs.ac.in (B. Mahato), ng13@iitbbs.ac.in (G. Naveen), bhumkar@iitbbs.ac.in (Y. G. Bhumkar) 
as flow past aircraft wings and propeller blades, high-rise buildings, bridge supports, power transmission lines and heat exchanger tubes. Engineers are usually concerned about the flow dynamics and unsteady forces acting on the bodies caused by the fluid motion. Famous collapse of the Tacoma Narrows Bridge attracted significant attention towards vortex induced instabilities, flow induced vibrations and aerodynamic flutter. Apart from imparting unsteady forces to the immersed bodies, time varying nature of the fluid flow also gives rise to sound generation and is often a cause of concern. For example, residents living close to windmill farms are exposed to aerodynamic noise caused by air flow over windmill blades. Thus, one needs to develop a detailed understanding for effects of incoming free-stream velocity and aspect ratio/shape of the body on the generated aeolian tones.

In one of the first experimental work on aeroacoustics, Strouhal [39] reported generation of aeolian tones for flow past a cylinder. Rayleigh [29] and Gerrard [8] subsequently established connection between the vortex shedding behind circular cylinder and radiated aeolian tones. Experiments associated with aeroacoustic problems face significant challenges due to presence of inherent background noise. Lighthill [19] proposed a theoretical approach to estimate aerodynamically generated sound with the help of an acoustic analogy [19,43-45] Some of the earlier research works $[2,6,26]$ used acoustic analogy to predict aeolian tones. Curle [2] further proposed modification in the Lighthill's acoustic analogy to include effects of solid boundaries on the aeroacoustic sound generation. At low Mach numbers, Curle [2] predicted that the dipole sound dominates over the quadrupole sound and the sound associated with the drag force has double the frequency compared to that associated with the lift force. It has been reported that the maximum amplitude of the radiated sound field is at right angles to the flow direction for such low Reynolds number flows past bluff bodies $[2,6,8,26]$ Using the similar acoustic analogy approach, Tam \& Hardin [40] and Wang et al. [42] estimated sound generated due to flow past a circular cylinder and a NACA-0012 aerofoil, respectively. Wang et al. [42] found that the quadrupole sound was significantly smaller as compared to that originated due to the lift and the drag dipoles for low Mach number flows. Researchers have also used an acoustic/viscous splitting approach $[9,35]$ to predict aeroacoustic noise generation at low Mach numbers. In this approach, flow quantities are decomposed into incompressible mean flow component and a perturbation about the mean. Mean flow quantities are first obtained by solving incompressible viscous flow equations and corresponding mean flow information is further used to solve the perturbation equations which provide acoustic quantities in the far field. Hardin \& Pope [9] and Shen \& Sorensen [35] predicted sound generation by a cylinder kept in an uniform flow using acoustic/viscous splitting method. In contrast to above two approaches, one can use an accurate but computationally expensive direct numerical simulation $(D N S)$ approach $[7,10,14-17,21]$ to predict flow induced sound field accurately. Using DNS approach, Inoue \& Hatakeyama [16] computed sound radiated by flow past a circular cylinder in the laminar flow regime, while Mahato et al. [21] computed sound radiated by an equilateral triangular wedge subjected to laminar flows at different angle of attacks. Computations based on the DNS ap- 
proach need to be carried out by highly accurate physical dispersion relation preserving schemes $[18,22,31]$. Although superiority of compact schemes in resolving flow details is well known, Dipankar \& Sengupta [3] pointed out the unphysical bias/directionality introduced by the compact schemes in the boundary and near-boundary nodes while performing simulations. Dipankar \& Sengupta [3] further recommended use of symmetrization technique to obtain similar nodal numerical properties at the inflow and outflow regions. While performing simulations, one has to be careful about unphysical bias introduced by the discretization schemes.

In the past, flow past elliptic cylinders at different free-stream conditions have been analyzed [24, 36, 38]. Shintani et al. [36] obtained detailed description of the low Reynolds number flow field near an elliptic cylinder by paying special attention towards the variation of aspect ratio of the cylinder and corresponding changes in the inertial effects of the fluid. Mittal \& Balachandar [24] performed numerical study to analyze two and three dimensional flow past elliptic cylinders of different aspect ratios. Mittal \& Balachandar [24] used a spectral collocation technique to simulate the full three-dimensional incompressible flow over elliptic cylinders to study unsteady separation and wake structures. Soumya \& Prakash [38] numerically studied effects of introduction of splitter plate on wake modifications of elliptic cylinders of different aspect ratios using finite element method.

Although many studies in the past have been devoted towards understanding flow past elliptic cylinders, modifications in the flow induced sound field due to change in aspect ratio of the cylinder has not received sufficient attention. It is expected that the aspect ratio of the elliptical cylinder will modify strength and convecting speed of sound sources in the wake region. One of the motivation behind the present work is to understand nature of acoustic sources in the wake region. Present work is based on the DNS approach which helps to obtain sound pressure variation in the domain [16] without any modeling. Solutions obtained using such accurate approach provides information about sound sources as well. Present manuscript focuses on following important points.

1. According to Powell's analogy [27], vorticity generated near the surface of a cylinder is related to the generation and propagation of sound pressure fluctuations. To extract information about equivalent noise sources, the vorticity based source term of Powell's equation has been evaluated and the variation of source intensity with aspect ratio of elliptic cylinder and free-stream Reynolds number has been analyzed.

2. Curle's acoustic analogy relates unsteady surface dipole sources created by the time varying forces acting on the body to the noise radiated in the far field [2]. Thus, the amplitudes and frequencies associated with fluctuating lift and drag forces have been analyzed for different cases.

3. To understand the modifications in the equivalent aeroacoustic noise source characteristics due to change in aspect ratio of the elliptic cylinder as well as Reynolds number, information about the sound sources has been derived by evaluating 
source term of Lighthill's equation using approximated Lighthill's stress tensor (ALST) term [1,19]. Derived information about equivalent noise sources has been correlated with the obtained disturbance pressure fields.

4. Proper orthogonal decomposition $(P O D)[33,34,37]$ of the disturbance pressure field has been performed to identify and quantify various dominant modes associated with radiated sound fields.

5. Accurate solution of compressible Navier-Stokes equations contains information about the hydrodynamic, acoustic and thermal modes together. These modes have been separated out using Doak's decomposition technique [41] to investigate the role of hydrodynamic fluctuations in dictating the characteristics of radiated sound field.

Present manuscript provides information about the mathematical formulation and numerical procedure in Section 2. Various numerical results discussing flow field and flow induced sound field are discussed in Section 3. Summary of the results and conclusions are given in Section 4.

\section{Problem definition}

Fig. 1 shows the schematic of uniform fluid flow over an elliptic cylinder at an angle of attack $(A O A)=4^{\circ}$ with Mach number $M=0.2$. Center of the elliptic cylinder has been located at the origin. Here, $r$ and $\theta$ provide the radial distance of a point in the flow field from center of the ellipse and the corresponding angle made by the radial vector with the negative $x$-axis in clockwise direction as shown in Fig. 1, respectively. The aspect ratio $(A R)$ of an elliptic cylinder is given as $A R=b / a$, where $a$ and $b$ are the lengths of the major and minor axes of elliptic cylinder, respectively. In the present computations, the length of the major axis is fixed as unity and the value of $b$

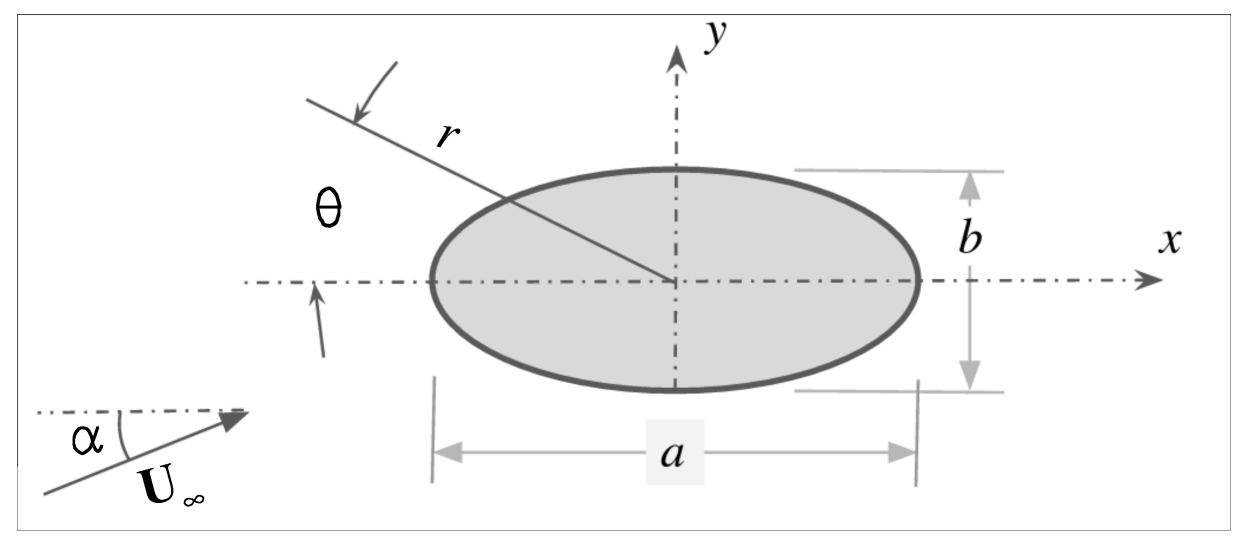

Figure 1: Schematic for uniform flow over an elliptic cylinder at an angle of attack $(A O A)=4^{\circ}$. 
is varied. The free-stream values of density $\left(\rho_{\infty}\right)$, temperature $\left(T_{\infty}\right)$, Prandtl number $\left(P r_{\infty}\right)$ and the ratio of specific heats $(\gamma)$ are prescribed as $1.12 \mathrm{~kg} / \mathrm{m}^{3}, 287 \mathrm{~K}, 0.7$ and 1.4 , respectively. Variation of the properties like thermal conductivity and molecular viscosity due to the change in temperature has been neglected at this relatively low Mach number $[16,21]$.

Simulations are performed for five different values of the aspect ratio $A R=0.6$, $0.7,0.8,0.9$ and 1.0. Among the considered aspect ratios, a case with $A R=1$ represents flow past a circular cylinder and displays bluff body behavior, while the case with $A R=0.6$ comparatively displays the nature of a streamlined body. Reynolds number is defined as $R e=\left(\rho_{\infty} U_{\infty} a\right) / \mu_{\infty}$. Here, $U_{\infty}$ and $\mu_{\infty}$ account for free-stream velocity and viscosity of the fluid, respectively. The effect of Reynolds number $(R e)$ on the generated sound fields has also been analyzed for five different Reynolds numbers $R e=100$, $120,130,150$, and 160 . The characteristics of flow past a circular cylinder $A R=1$ are known to be two-dimensional for the chosen values of Reynolds number [47]. Hence, two-dimensional simulations are carried out in the present work.

\subsection{Numerical methodology, grid and computational domain details}

The present approach is based on the DNS methodology where the unsteady compressible mass, momentum and energy conservation equations for fluid flow are solved in a conservative form $[11,21]$. The governing equations and flow parameters are non-dimensionalized using free-stream properties of fluid flow. The density, pressure, viscosity, and temperature are non-dimensionalized using the free-stream parameters $\rho_{\infty}, \rho_{\infty} U_{\infty}^{2}, \mu_{\infty}$ and $T_{\infty}$, respectively. The lengths $(x, y$, and $r$ ), velocity components, and time are scaled by the terms $D, U_{\infty}$, and $D / U_{\infty}$, respectively. Further details on governing equations and their non-dimensionalized form have been adequately explained in the previous work [21] and are not provided here to avoid repetition. From here, all the parameters are considered to be in non-dimensional form. Computations have been performed in an $O$-grid domain with a radius $r=1500$ with an elliptic cylinder kept at the center of the domain. Using elliptic grid generation method [11], we have constructed body fitted structured grid around the cylinder with 501 grid points in the azimuthal direction and 900 points in the radial (wall normal) direction.

Inoue \& Hatakeyama [16] provided a strategy to distribute the grid points in the wall normal direction while performing direct computation of noise radiation from a circular cylinder kept in a uniform free-stream at $R e=150$ and $M=0.2$. Based on the required grid spacing in the wall normal direction, Inoue \& Hatakeyama [16] divided the computational domain into three parts for resolving the boundary layer, sound disturbances and to dissipate flow and sound disturbances towards outer boundary of the domain. We have followed a similar methodology for distributing grid points in the wall normal direction. The first grid point in the wall normal direction is kept at a distance of $r=0.004$ from the surface and there are around 15 points in the boundary layer. Grid spacing in the sound region $[0.5 \leq r \leq 100]$ increases smoothly away from the surface of elliptic cylinder with maximum grid spacing maintained as $\Delta r_{\max }=$ 


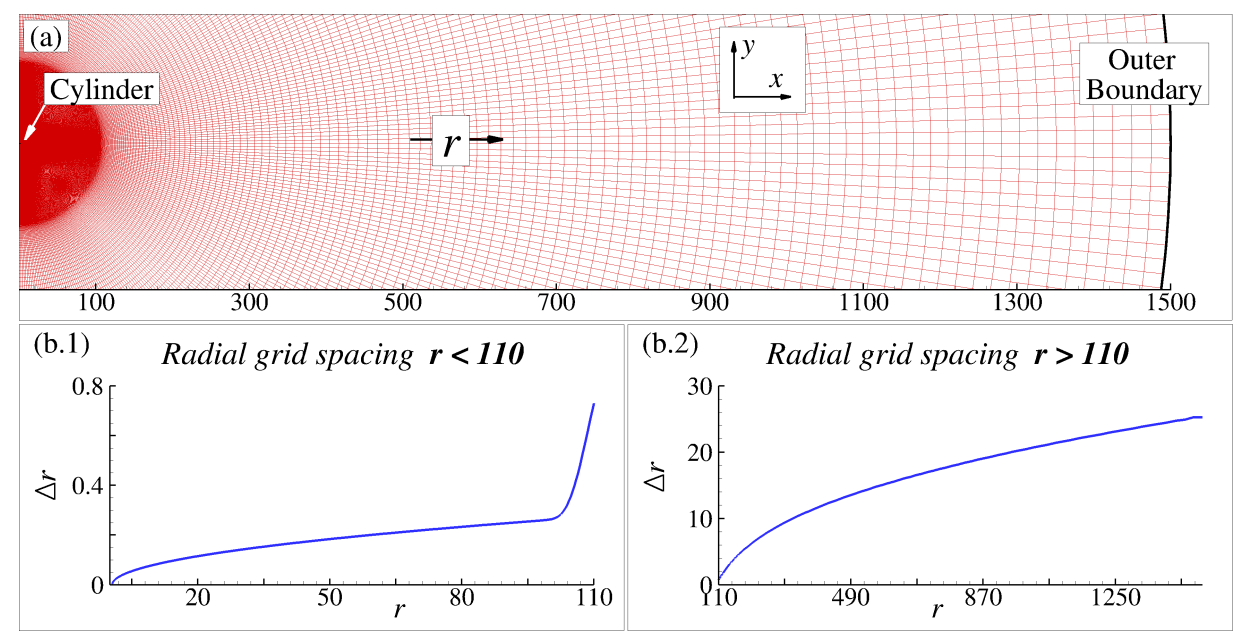

Figure 2: (a) Distribution of grid points along the wall normal direction. (b) Variation of grid spacing $(\Delta r)$ with radial distance $r$ in sound and buffer zones.

0.25 in the sound zone. Thus there are around 750 grid points in the wall normal direction in the sound region while rest of the grid points are coarsely distributed in the buffer region $[100 \leq r \leq 1500]$ which is used to further dissipate the flow and sound disturbances. A typical grid point distribution in the wall normal direction has been shown in Fig. 2(a). Distance between successive grid points along radial direction is displayed in Fig. 2(b). One can observe that the grid points are closely spaced near the cylinder surface while they are coarsely distributed towards the outer boundary of the domain and variation of grid spacing across different regions in the wall normal direction is smooth.

On the surface of cylinder, no-slip and adiabatic boundary conditions are prescribed. Characteristic based boundary conditions are implemented at the far-field boundary. The convective derivative terms have been discretized using optimized coupled compact difference $(O C C D)$ scheme $[21,22]$, while time integrations have been performed using spectrally optimized five stage Runge-Kutta (ORK5) scheme [28]. The second order derivative terms in the governing partial differential equations are approximated using explicit second order central finite difference method. A compressible flow solver based on a parallel computing framework and above discretization schemes has been developed.

\section{Results and discussion}

Simulations for flow past elliptic cylinders of different aspect ratios have been performed for five different Reynolds number cases. Angle of attack has been maintained as $4^{\circ}$ in all the simulations. Computations have been performed for sufficiently long duration so that the flow reaches its quasi-steady state. Time averaged mean flow field, 


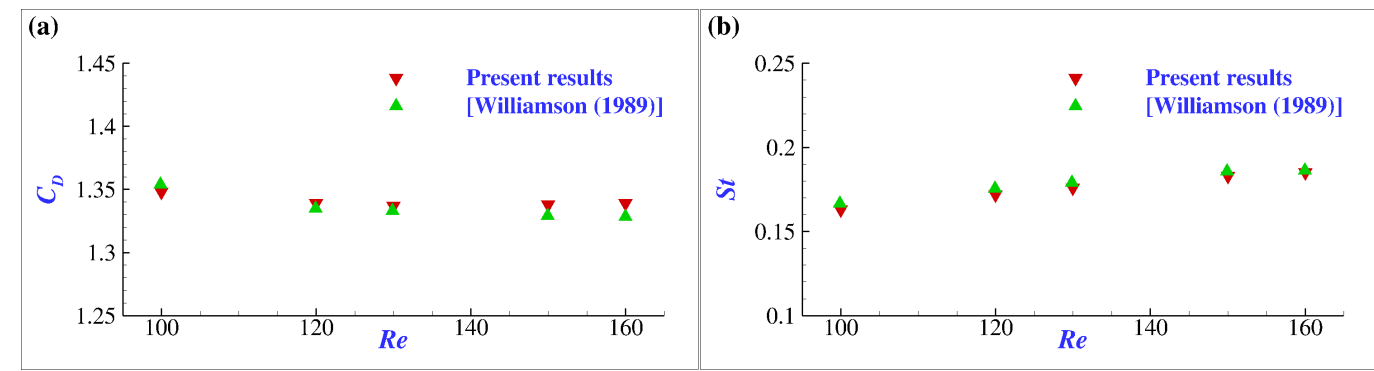

Figure 3: Validation of present numerical results obtained for flow over a circular cylinder $A R=1.0$ are shown here. Frames (a) and (b) show the variation of mean drag coefficient $C_{D}$ and Strouhal number $S t$, respectively for all indicated values of $R e$. The present numerical results are in good agreement with those of Williamson [46].

the mean lift and drag coefficients as well as disturbance pressure fields have been calculated by considering time averaging of computed solution over 20 lift cycles. As shedding process has not been observed for the case with $A R=0.6 \& R e=100$, mean and disturbance properties are not shown in the results.

For flow over a circular cylinder case $(A R=1.0)$, the mean drag coefficient $\left(C_{D}\right)$ and the Strouhal number $(S t)$ obtained from the present simulations are shown in Fig. 3(a) and Fig. 3(b), respectively for indicated values of Re. It is observed that the present results $\left(C_{D}\right.$ and $\left.S t\right)$ are in good agreement with the experimental results of Williamson [46]. This proves the validation of present methodology and the DNS solver. A detailed validation and verification studies are reported in our previous research works $[7,21,22]$.

Fig. 4 shows the variation of the mean and the disturbance properties of computed flow fields for different values of Reynolds number and aspect ratio. Figs. 4(a) and (b) represent variation of the mean lift $\left(C_{L}\right)$ and drag $\left(C_{D}\right)$ coefficients, respectively. For the lower values of aspect ratio, there is a higher mean lift coefficient due to positive angle of attack. As aspect ratio increases, elliptic cylinder transforms to a circular cylinder providing zero mean lift coefficient while the drag coefficient increases with aspect ratio as the cylinder displays a bluff body nature at higher aspect ratios. As the viscous effects are dominant in the considered Reynolds number range, higher mean drag coefficient is observed at lower Reynolds number.

Fluctuations in the lift $\left(l^{\prime}\right)$ and the drag $\left(d^{\prime}\right)$ coefficients are evaluated from the mean $\&$ the instantaneous values of lift and drag coefficients, respectively. Variations of root mean square values $(R M S)$ of fluctuations in the lift and the drag coefficients $\left(l_{R M S}^{\prime} \& d_{R M S}^{\prime}\right)$ with aspect ratio for different values of Reynolds number are displayed in Figs. 4(c) and (d), respectively. For a given Reynolds number, magnitudes of $l_{R M S}^{\prime}$ and $d_{R M S}^{\prime}$ increase with increase in aspect ratio due to increase in the strength of shed vortex with aspect ratio. As the strength of shed vortex increases with aspect ratio, the fluctuating amplitudes of $l^{\prime}$ and $d^{\prime}$ are also increased. The effect of Reynolds number on $l^{\prime}$ and $d^{\prime}$ is also similar. Fig. 4(e) displays the effect of aspect ratio on Strouhal number 

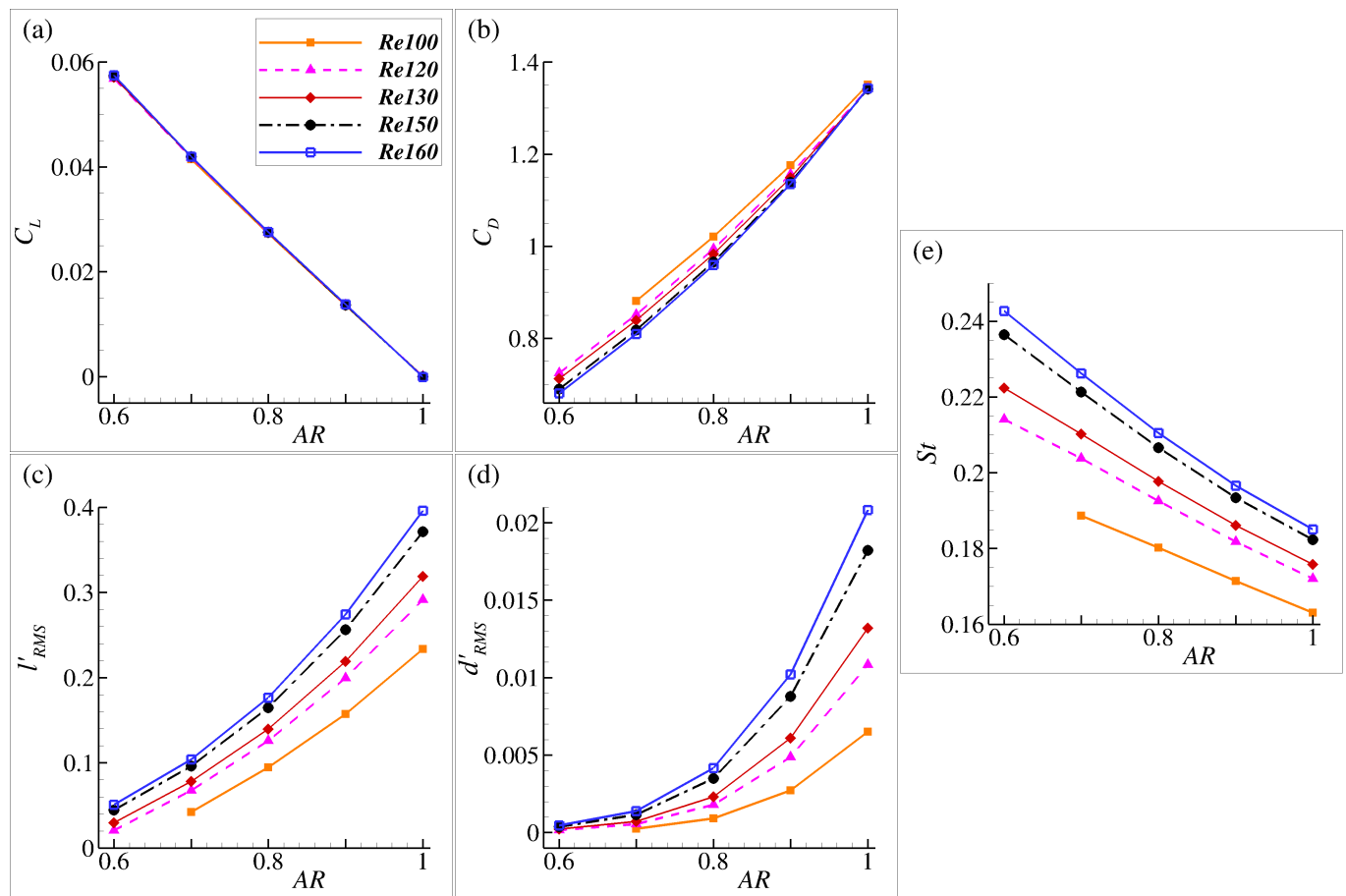

Figure 4: Variation of the flow field parameters with aspect ratio $(A R)$ for indicated values of Reynolds number. Frames (a) and (b) represent the variation of the mean lift and the drag coefficients, respectively. Frames (c) and (d) show the variation of the $R M S$ values for fluctuating lift and drag coefficients, respectively. Frame (e) displays the variation of Strouhal number $(S t)$ with aspect ratio.

$S t$ for indicated values of Reynolds number. For a given Reynolds number, the value of Strouhal number $S t$ decreases with increase in aspect ratio. On the other hand, Strouhal number increases with increase in Reynolds number for all values of aspect ratio.

Simulation results for the case of flow past an elliptic cylinder with $A R=0.8$ and $R e=130$ have been shown in Fig. 5. Fig. 5(a) shows variation of the fluctuating lift and the drag coefficients with time. Transient behavior of vortex shedding pattern has been shown in Fig. 5(b) by plotting vorticity $\left(\omega_{z}\right)$ contours over a one periodic cycle of the lift coefficient at identified instants. Results show formation of the Von Kármán vortex street with vortices being periodically shed into the wake region. Positive and negative values of vorticity are denoted as solid and dashed lines, respectively. Respective instants in Fig. 5(b) are identified with filled circles in Fig. 5(a). One can observe that the frequency of the drag coefficient is two times higher than that of the lift coefficient. Wake features are accurately resolved over long radial distances.

Disturbance pressure field $\left(p^{\prime}(x, y, t)\right)$ at any instant has been evaluated from the instantaneous pressure field and the mean pressure field as given by,

$$
p^{\prime}(x, y, t)=p(x, y, t)-p_{m}(x, y) .
$$




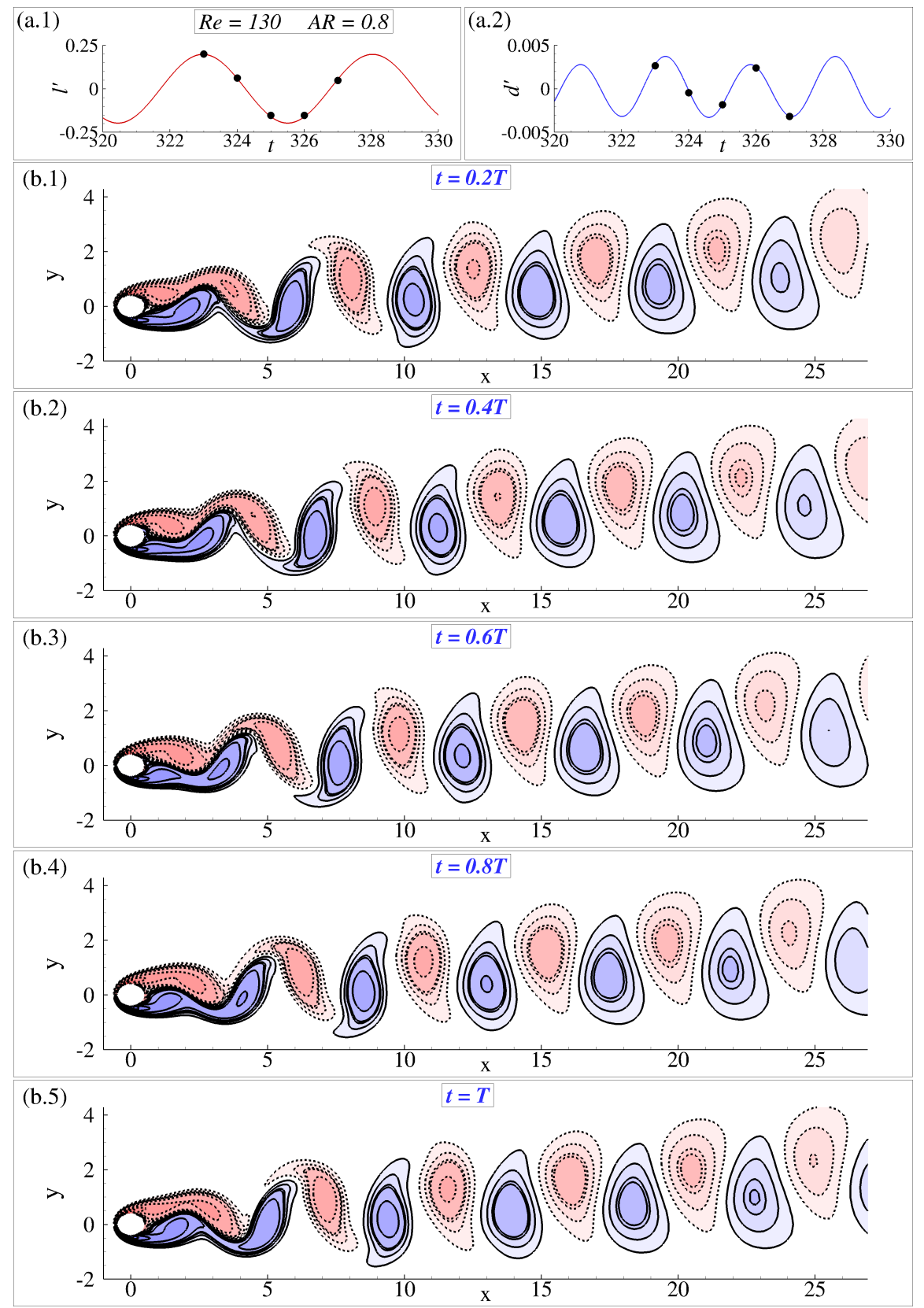

Figure 5: Evolution of Von Kármán vortex street for one complete lift cycle. (a.1) and (a.2) are the fluctuating lift and drag variation with time, respectively. Black dot represents corresponding time instant of vorticity plot. (b) Vorticity $\left(\omega_{z}\right)$ variation in the wake for one lift cycle.

Fig. 6 displays disturbance pressure fields at indicated instants over one vortex shedding cycle for flow over an elliptic cylinder with $A R=0.8$ at $R e=130$. Shedding of 


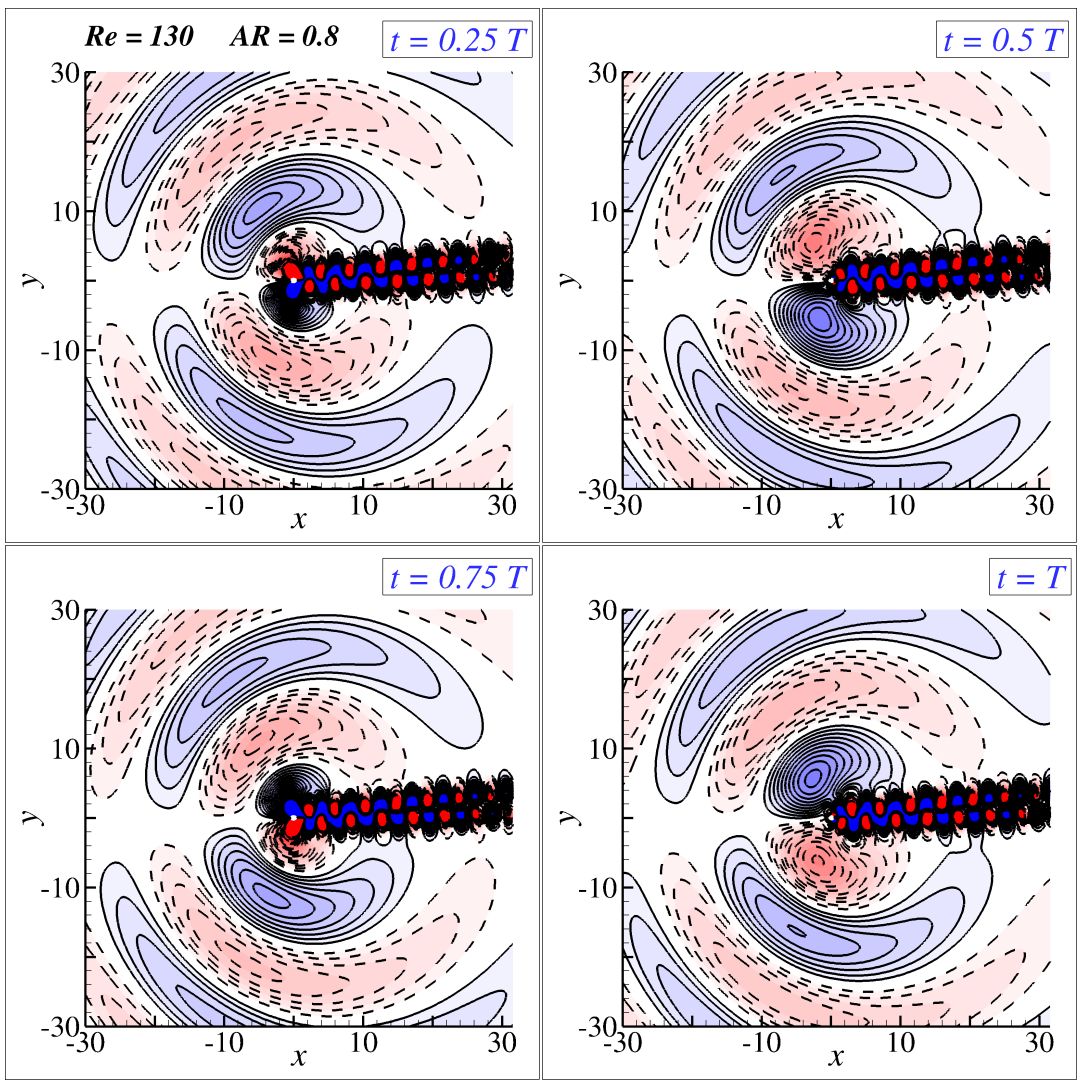

Figure 6: Transient evolution and propagation of disturbance pressure pulses from the top and the bottom surfaces of an elliptic cylinder with $A R=0.8$ and $R e=130$ over one shedding cycle. Contour levels vary from -0.01 to 0.01 with 40 equi-spaced sub-levels.

vortices from the top and the bottom surfaces has resulted in generation of disturbance pressure pulses. These pulses propagate away from the cylinder as time progresses. Solid and dashed lines represent compression and rarefaction regions of generated sound field. Thus generated sound fields are characterized by nature and strength of vortex shedding patterns.

Next, we analyze the properties of equivalent noise source mechanisms associated with acoustic analogies $[1,2,19,27]$ and relate them with radiated sound field characteristics.

\subsection{Acoustic analogies}

Lighthill [19] considered mass and momentum conservation equations of the fluid flow to derive a single second order partial differential equation in space and time whose left hand side represents the wave motion while the right hand side provides the information about equivalent sound source terms. Lighthill's [19] equation in terms of 
the fluctuating component of the pressure $\left(p^{\prime}\right)$ is given as $[16,19]$,

$$
\frac{1}{c_{\infty}^{2}} \frac{\partial^{2} p^{\prime}}{\partial t^{2}}-\nabla^{2} p^{\prime}=\frac{\partial^{2} T_{i j}}{\partial x_{i} \partial x_{j}} .
$$

Here, $c_{\infty}$ is the speed of sound. The term $T_{i j}$ indicates Lighthill stress tensor and is given as, $T_{i j}=\rho u_{i} u_{j}+\left(p^{\prime}-c_{\infty}^{2} \rho^{\prime}\right) \delta_{i j}-\tau_{i j}$, where $\tau_{i j}$ is the viscous stress tensor. Thus the wave equation proposed by Lighthill is inhomogeneous equation with the equivalent source term on the right hand side. Note that the equations and other parameters are in dimensional form. For an isentropic flow assumption $\left(p^{\prime}=c_{\infty}^{2} \rho^{\prime}\right)$ with a compact source region and neglecting the viscous stress tensor $\left(\tau_{i j}=0\right)$, the Lighthill's stress tensor has been approximated as $T_{i j}=\rho u_{i} u_{j}$ and is called as approximated Lighthill's stress tensor. Lighthill assumed that the sound is radiated into free-space and there by neglecting the effect of scattering. The approximate solution to the Lighthill's equation (Eq. (3.2)) is expressed as $[2,16]$,

$$
p^{\prime}(\mathbf{x}, t)=\frac{x_{i} x_{j}}{2^{3 / 2} \pi c_{\infty}^{3 / 2}|\mathbf{x}|^{5 / 2}} \int_{-\infty}^{\tau}\left[\frac{\partial^{2}}{\partial t^{\prime 2}} \int_{V} T_{i j}\left(\mathbf{y}, t^{\prime}\right) \mathrm{d} \mathbf{y}\right] \frac{\mathrm{d} t^{\prime}}{\sqrt{\tau-t^{\prime}}},
$$

where $\tau=t-|\mathbf{x}| / c_{\infty}$ denotes the retarded time with $\mathbf{x}$ being the position vector of the observer. The right-side denotes the volume distribution of equivalent quadrupole sources. Next, Curle [2] extended the Lighthill's theory by including the effects of solid boundaries and obtained the solution to Eq. $(3.2)$ as $[2,16]$,

$$
\begin{aligned}
p^{\prime}(\mathbf{x}, t)= & \frac{x_{i} x_{j}}{2^{3 / 2} \pi c_{\infty}^{3 / 2}|\mathbf{x}|^{5 / 2}} \int_{-\infty}^{\tau}\left[\frac{\partial^{2}}{\partial t^{\prime 2}} \int_{V} T_{i j}\left(\mathbf{y}, t^{\prime}\right) \mathrm{d} \mathbf{y}\right] \frac{\mathrm{d} t^{\prime}}{\sqrt{\tau-t^{\prime}}} \\
& \quad \frac{x_{i}}{2^{3 / 2} \pi c_{\infty}^{1 / 2}|\mathbf{x}|^{3 / 2}} \int_{-\infty}^{\tau}\left[\frac{\partial}{\partial t^{\prime}} \oint_{S} f_{i}\left(\mathbf{y}, t^{\prime}\right) \mathrm{d} \ell(\mathbf{y})\right] \frac{\mathrm{d} t^{\prime}}{\sqrt{\tau-t^{\prime}}}
\end{aligned}
$$

The force per unit length $(d \ell)$ on the surface of boundary is denoted by $f_{i}$. The first and the second terms on the right-hand side of Eq. (3.4) represent the equivalent sources associated with quadrupole and dipole sound fields, respectively.

Sengupta et al. [32] suggested an alternate way to look at the pressure field in terms of total mechanical energy (or total pressure) while discussing vortex induced instability in an incompressible wall-bounded shear layer. The total mechanical energy $\left(E=p / \rho+U^{2} / 2\right)$ was related to rotationality of the flow [32]. Powell [27] proposed an approximate version of Lighthill's analogy [19] in terms of rotationality of the flow. Powell further assumed the fluid to be incompressible inside the source region and then expressed the Lighthill's equation (Eq. (3.2)) in terms of vorticity vector $(\omega)$ as [27],

$$
\begin{aligned}
& \frac{1}{c_{\infty}^{2}} \frac{\partial^{2} p^{\prime}}{\partial t^{2}}-\frac{\partial^{2} p^{\prime}}{\partial x_{i}^{2}} \\
= & \nabla \cdot\left[\rho(\omega \times \mathbf{v})+\nabla\left(\frac{1}{2} \rho|\mathbf{v}|^{2}\right)-\mathbf{v} \frac{\partial \rho}{\partial t}-\frac{1}{2}|\mathbf{v}|^{2} \nabla \rho\right]+\frac{\partial^{2}}{\partial t^{2}}\left(\frac{p^{\prime}}{c_{\infty}^{2}}-\rho^{\prime}\right),
\end{aligned}
$$


where $\mathbf{v}$ denotes the velocity vector.

Assuming the flow to be isentropic and the fluid is incompressible in the source region, Eq. (3.5) is further reduced to

$$
\frac{1}{c_{\infty}^{2}} \frac{\partial^{2} p^{\prime}}{\partial t^{2}}-\frac{\partial^{2} p^{\prime}}{\partial x_{i}^{2}}=\nabla \cdot\left[\rho(\omega \times \mathbf{v})+\nabla\left(\frac{1}{2} \rho|\mathbf{v}|^{2}\right)\right] .
$$

Next, we evaluate the information about equivalent sound sources derived from the above mentioned acoustic analogies for various cases investigated in the present work before discussing the results for sound pressure and sound power.

\subsection{Equivalent sound sources}

Let us first look at the Powell's analogy by considering Eq. (3.6). As some studies in the past had highlighted that the flow induced sound was mainly due to the vorticity effects in the source region $[13,20,25,27]$, the contribution of the second term on the right-hand side of Eq. (3.6) towards the sound production is neglected in the present study. The far-field sound pressure is governed by the equation in [27],

$$
\frac{1}{c_{\infty}^{2}} \frac{\partial^{2} p^{\prime}}{\partial t^{2}}-\frac{\partial^{2} p^{\prime}}{\partial x_{i}^{2}}=\rho \nabla \cdot(\omega \times \mathbf{u})
$$

where, $\mathbf{u}$ is disturbance velocity vector. Thus, the vorticity generated by the application of the no-slip boundary condition at the cylinder surface generates sound pressure fluctuations in the domain according to the Powell's [27] vortex sound theory. The right hand side term in Eq. (3.7), has been evaluated for different Reynolds number and aspect ratio cases.

Fig. 7 shows intensity of Powell's source term $(\nabla \cdot(\boldsymbol{\omega} \times \mathbf{u}))$ in the cylinder wake for indicated values of Reynolds number at $A R=0.8$. The strength of the noise source term has been increased with increase in Reynolds number due to increase in strength of vorticity. Hence, minimum and maximum intensities of noise source term are observed at $R e=100$ and $R e=160$, respectively. Consequently, the generated sound field must show relatively higher intensity at $R e=160$ than at $R e=100$ and this has been confirmed later using sound field parameters (net radiated sound power and directivity patterns).

Fig. 8 represents variation of Powell's source intensity with aspect ratio of elliptic cylinder at $R e=130$. One observes equivalent noise source term intensity increases with aspect ratio such that minimum and maximum values of intensity are observed at $A R=0.6$ and $A R=1.0$, respectively. The shed vortex from an elliptic cylinder with $A R=0.6$ has relatively lower strength than the shed vortex from the cylinder with $A R=1.0$. This is due to the fact that the cylinder with $A R=0.6$ displays a behavior similar to a streamline body while the cylinder with $A R=1.0$ exhibits the behavior of bluff body. Therefore, the sound field intensity must be comparatively higher at $A R=1.0$ than at $A R=0.6$ (as shown later). 

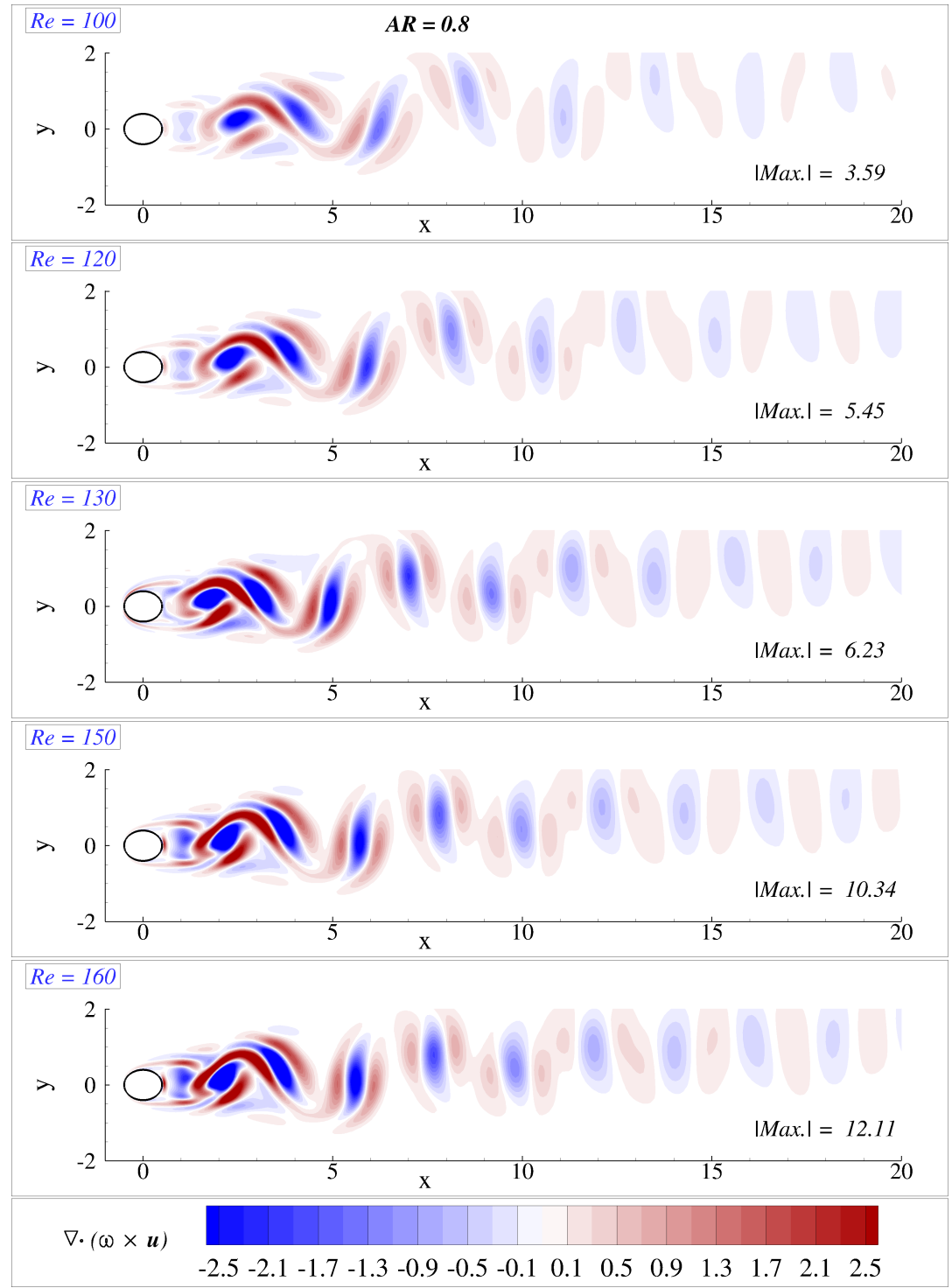

Figure 7: Variation in intensity of Powell's source term $\nabla \cdot(\boldsymbol{\omega} \times \mathbf{u})$ with Reynolds number for $A R=0.8$.

For flow past bluff bodies at relatively low Mach numbers, the equivalent quadrupole sound sources are negligible [16]. For a two dimensional flow past a rigid body with compact equivalent sound source region, one can further simplify the Curle's solution given by Eq. (3.4) as [30],

$$
p^{\prime}(\mathbf{x}, t)=\frac{1}{4 \pi c_{\infty}} \frac{x_{i}}{|\mathbf{x}|^{2}}\left[\frac{\partial}{\partial t^{\prime}} \oint_{S} f_{i}\left(\mathbf{y}, t^{\prime}\right) \mathrm{d} \ell(\mathbf{y})\right]=\frac{1}{4 \pi c_{\infty}} \frac{x_{i}}{r^{2}}\left[\frac{\partial F_{i}}{\partial t}\right]
$$

where, $F_{i}=\oint_{S} f_{i}\left(\mathbf{y}, t^{\prime}\right) \mathrm{d} \ell(\mathbf{y})$ is net force acting on the cylinder along $i^{\text {th }}$ direction 


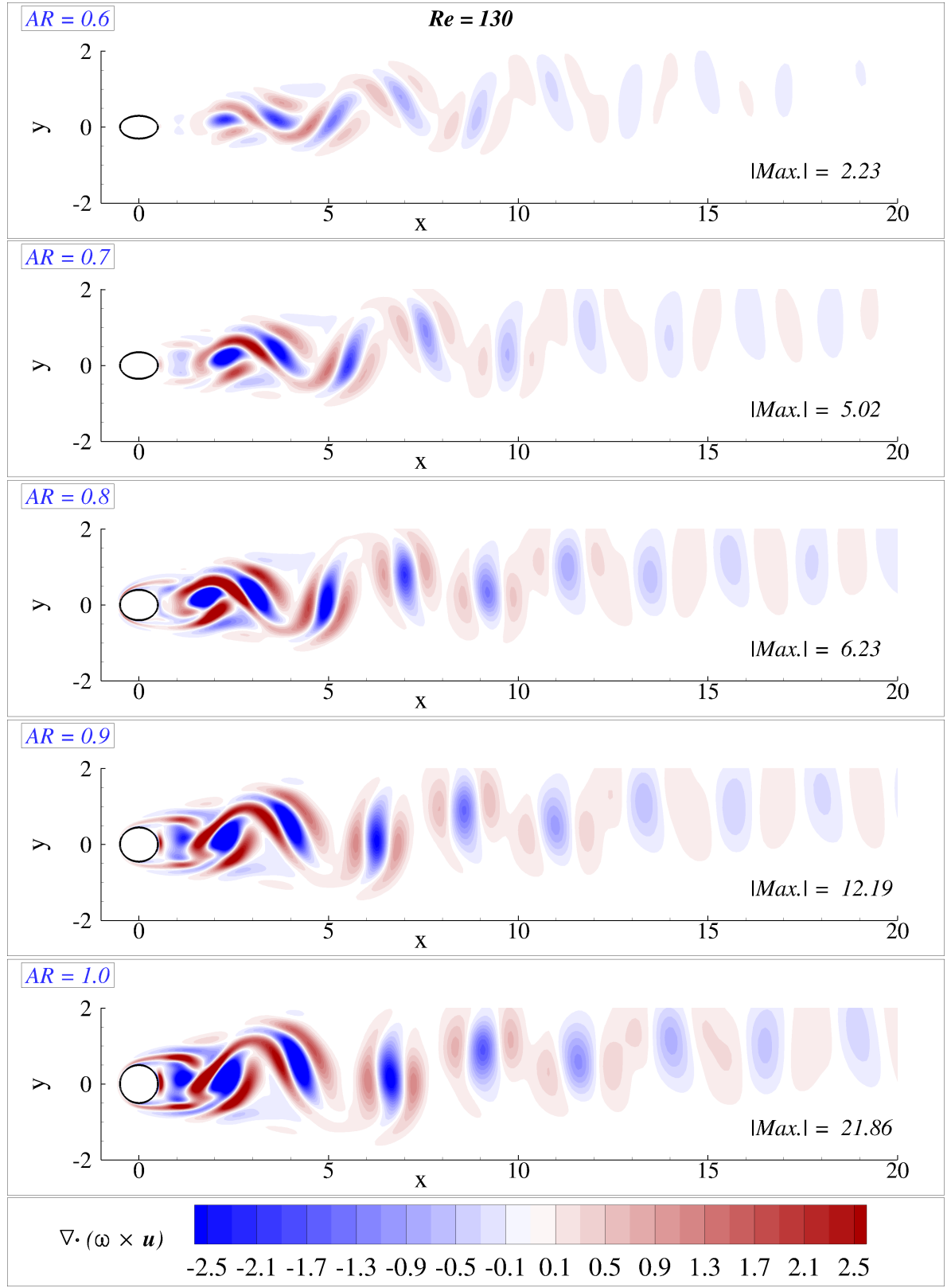

Figure 8: Variation in intensity of Powell's source term $\nabla \cdot(\boldsymbol{\omega} \times \mathbf{u})$ with aspect ratio for $R e=130$.

(either lift or drag forces) and the square bracket indicates derivative at retarded time. Eq. (3.8) suggests that the sound generated in the far-field depends on the time variation of the fluctuating loads acting on the body. Fluctuations in the lift and the drag coefficients caused by the periodic shedding of vortices from flow past 


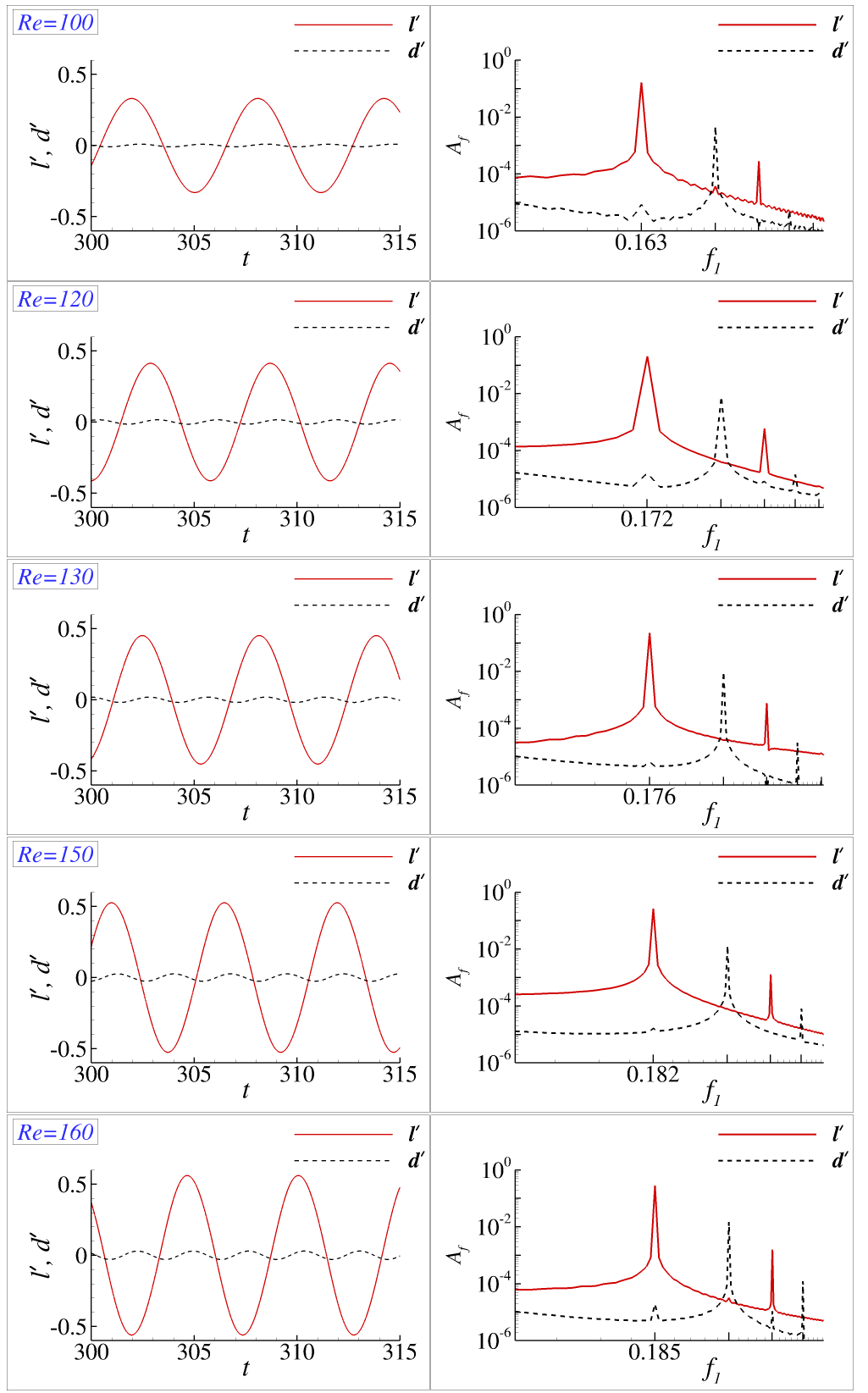

Figure 9: Time varying fluctuations in the lift \& the drag coefficients and their Fast Fourier Transforms have been shown for flow past a circular cylinder $(A R=1.0)$ at the indicated values of Reynolds number.

bluff bodies were related to the amplitude and frequency of sound waves by various researchers $[16,21,23]$. Information about the frequency and amplitude of the lift and the drag fluctuations dictates the sound field as given by the Curle's Eq. (3.8) in [30]. 


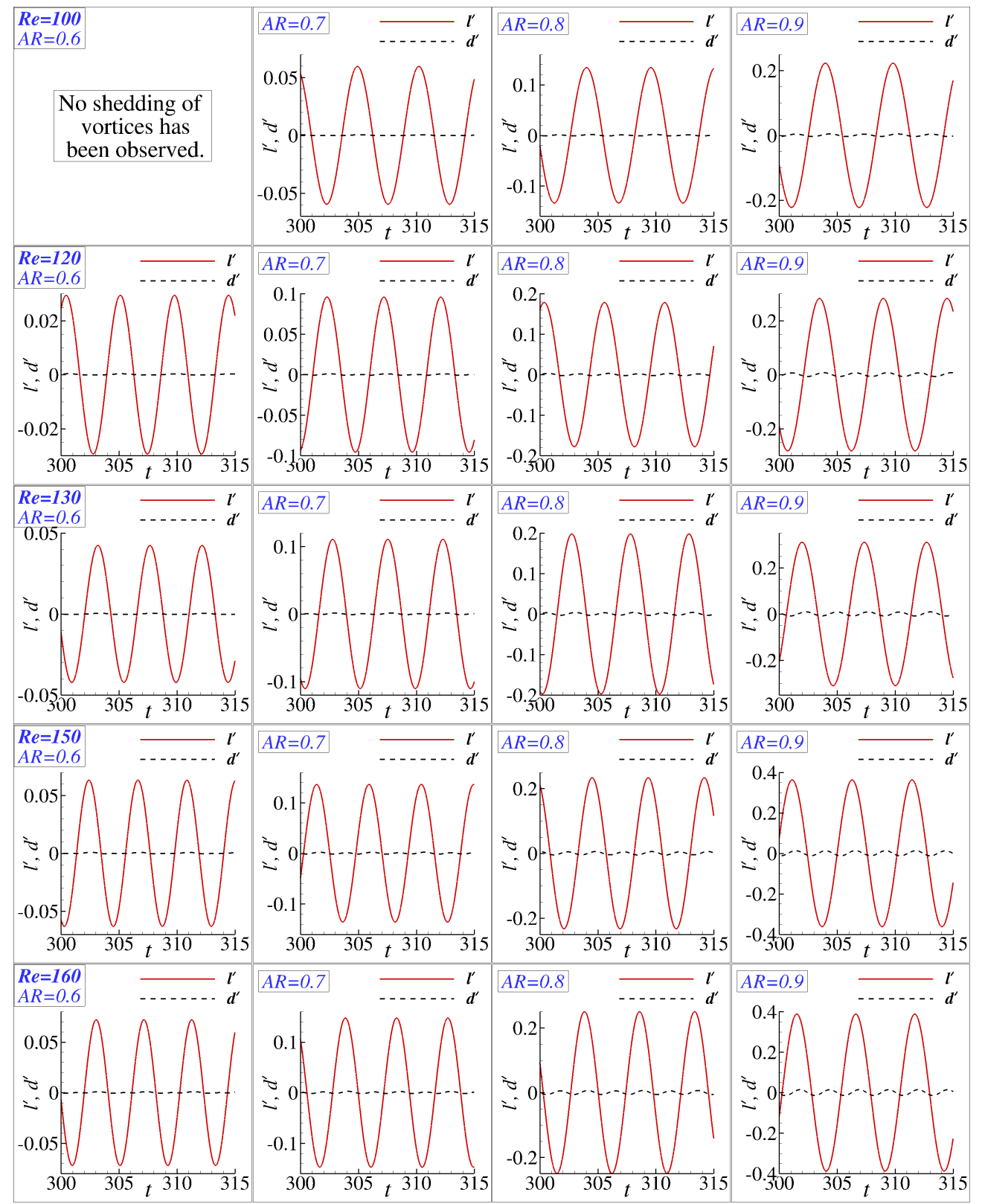

Figure 10: Variation of $l^{\prime}$ and $d^{\prime}$ with time has been shown for indicated values of aspect ratio and Reynolds number.

Fig. 9 displays variation of fluctuations in the lift $l^{\prime}$ and the drag $d^{\prime}$ coefficients and their frequency content for flow over a circular cylinder $A R=1.0$ at indicated values of Reynolds number. Left column shows variation of $l^{\prime}$ and $d^{\prime}$ with time whereas the right column represents corresponding frequency characteristics obtained from Fast Fourier 


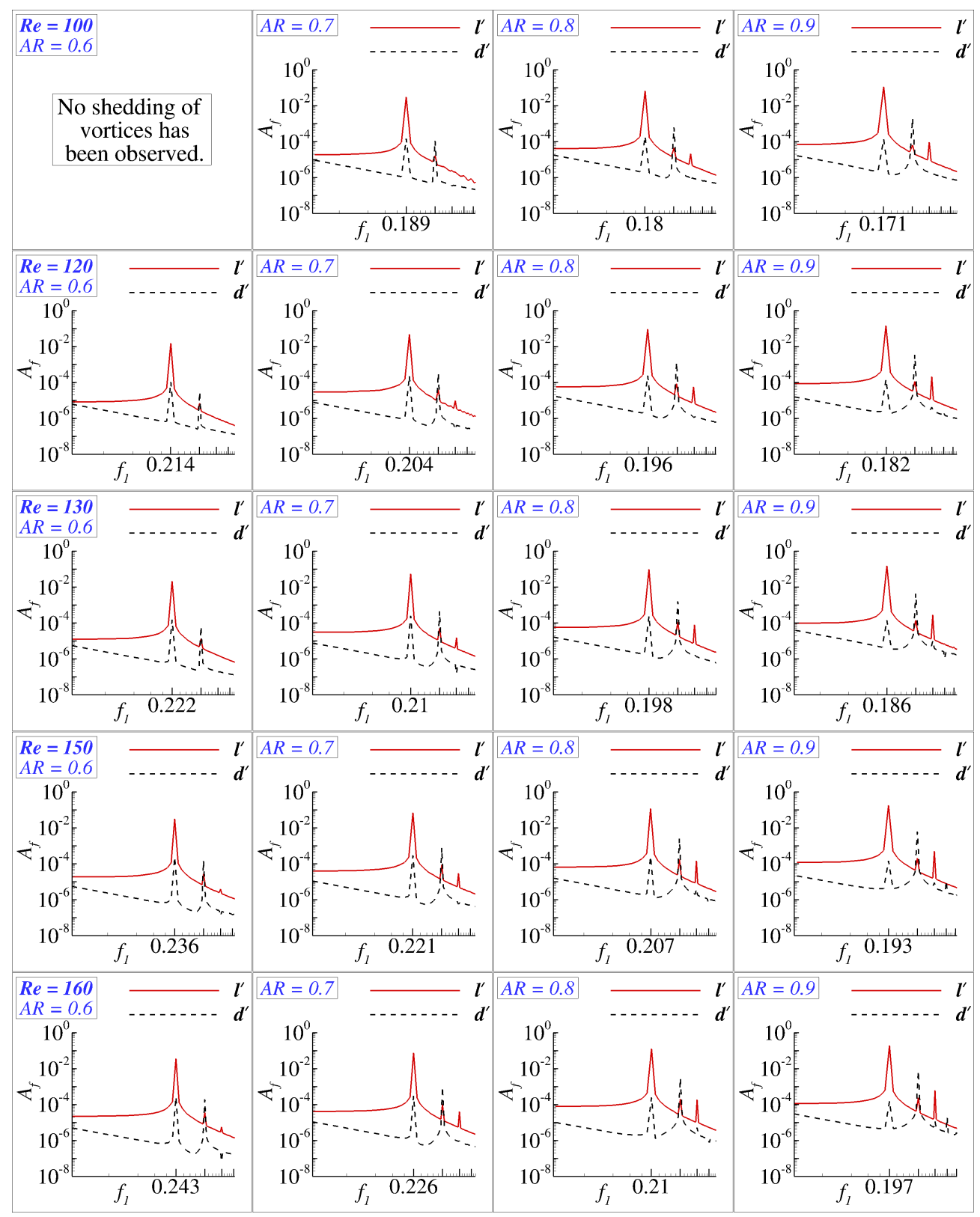

Figure 11: FFTs of time varying fluctuations in the lift and the drag coefficients are shown for indicated values of aspect ratio and Reynolds number. Both, $x$ and $y$-axis labels are plotted in logarithmic scale.

Transform $(F F T)$. The amplitude of $l^{\prime}$ is much higher compared to the amplitude of $d^{\prime}$ for all values of Reynolds number. Peaks of $l^{\prime}$ are identified at $f_{1}=f_{o} \& 3 f_{o}$ while the peaks of $d^{\prime}$ are identified at $f_{1}=2 f_{o} \& 4 f_{o}$. Here, $f_{o}$ is frequency of vortex shedding. Amplitudes of both $l^{\prime}$ and $d^{\prime}$ are found to be increasing with increase in 
Reynolds number. The resultant sound field intensity must also increase with increase in Reynolds number and it must be dominated by the lift dipole as lift fluctuations are more dominant compared to drag fluctuations.

Fig. 10 displays variation of $l^{\prime}$ and $d^{\prime}$ with time for indicated values of aspect ratio and Reynolds number. Vortex shedding has not been observed for $A R=0.6$ at $R e=$ 100. Aspect ratio and Reynolds number are increased from left to right and top to bottom, respectively. Amplitude of $d^{\prime}$ is significantly smaller than that of $l^{\prime}$ for all values of aspect ratio and Reynolds number. It is observed that amplitudes of $l^{\prime}$ and $d^{\prime}$ are found to be increasing with increase in Reynolds number as well as aspect ratio of elliptic cylinder.

Fig. 11 shows frequencies and amplitudes associated with the lift and the drag fluctuations for different values of aspect ratio and Reynolds number. Peaks of both $l^{\prime}$ $\& d^{\prime}$ are identified at shedding frequency $f_{o}$ and its higher harmonics $\left(2 f_{o}, 3 f_{o} \& 4 f_{o}\right)$, unlike the case with $A R=1.0$ where peaks of $l^{\prime}$ are identified at $f_{o} \& 3 f_{o}$ and those of $d^{\prime}$ are located at $2 f_{o} \& 4 f_{o}$. Vortex shedding frequency decreases as the aspect ratio increases for a particular Reynolds number. Decrease in vortex shedding frequency with the increase in aspect ratio has also been observed in Fig. 4(e).

From Figs. 9 and 10, one concludes that the fluctuations in the lift variation should contribute significantly higher to generated sound field as compared to the fluctuations in the drag coefficient. Frequency and the corresponding wavelength of the generated sound waves should be dictated by the variation of $l^{\prime}$.

Cheong et al. [1] obtained solutions for the incompressible flow past a circular cylinder at high Reynolds number $R e=1.58 \times 10^{4}$ and relied on hybrid $C A A$ method to simulate flow induced sound field. In this approach, Cheong et al. [1] have used the approximated form of the Lighthill's stress tensor $T_{i j}=\rho u_{i} u_{j}$ to prescribe equivalent sound source term for solving linearized Euler equations using high accuracy difference scheme. Let $u_{i}^{\prime}(\mathbf{x}, t)$ indicates the fluctuating component of the velocity field with $u_{i}(\mathbf{x}, t)$ and $\bar{u}_{i}(\mathbf{x})$ representing instantaneous and the mean components of the velocity field, respectively. The equivalent source term $\left(S_{i}=\frac{\partial T_{i j}}{\partial x_{j}}\right)$ has been modeled using approximated Lighthill's stress tensor $(A L S T)$ as [1],

$$
S_{A L S T, i}=-\frac{\partial \rho u_{i}^{\prime} u_{j}^{\prime}}{\partial x_{j}}-\frac{\partial \rho u_{i}^{\prime} \bar{u}_{j}}{\partial x_{j}}-\frac{\partial \rho \bar{u}_{i} u_{j}^{\prime}}{\partial x_{j}}-\frac{\partial \rho \bar{u}_{i} \bar{u}_{j}}{\partial x_{j}} .
$$

Eq. (3.9) is further decomposed into three separate terms as [1],

$$
\begin{aligned}
& S_{\text {self }, i}=-\frac{\partial \rho u_{i}^{\prime} u_{j}^{\prime}}{\partial x_{j}}, \\
& S_{\text {shear }, i}=-\frac{\partial \rho u_{i}^{\prime} \bar{u}_{j}}{\partial x_{j}}-\frac{\partial \rho \bar{u}_{i} u_{j}^{\prime}}{\partial x_{j}}, \\
& S_{0, i}=-\frac{\partial \rho \bar{u}_{i} \bar{u}_{j}}{\partial x_{j}}
\end{aligned}
$$




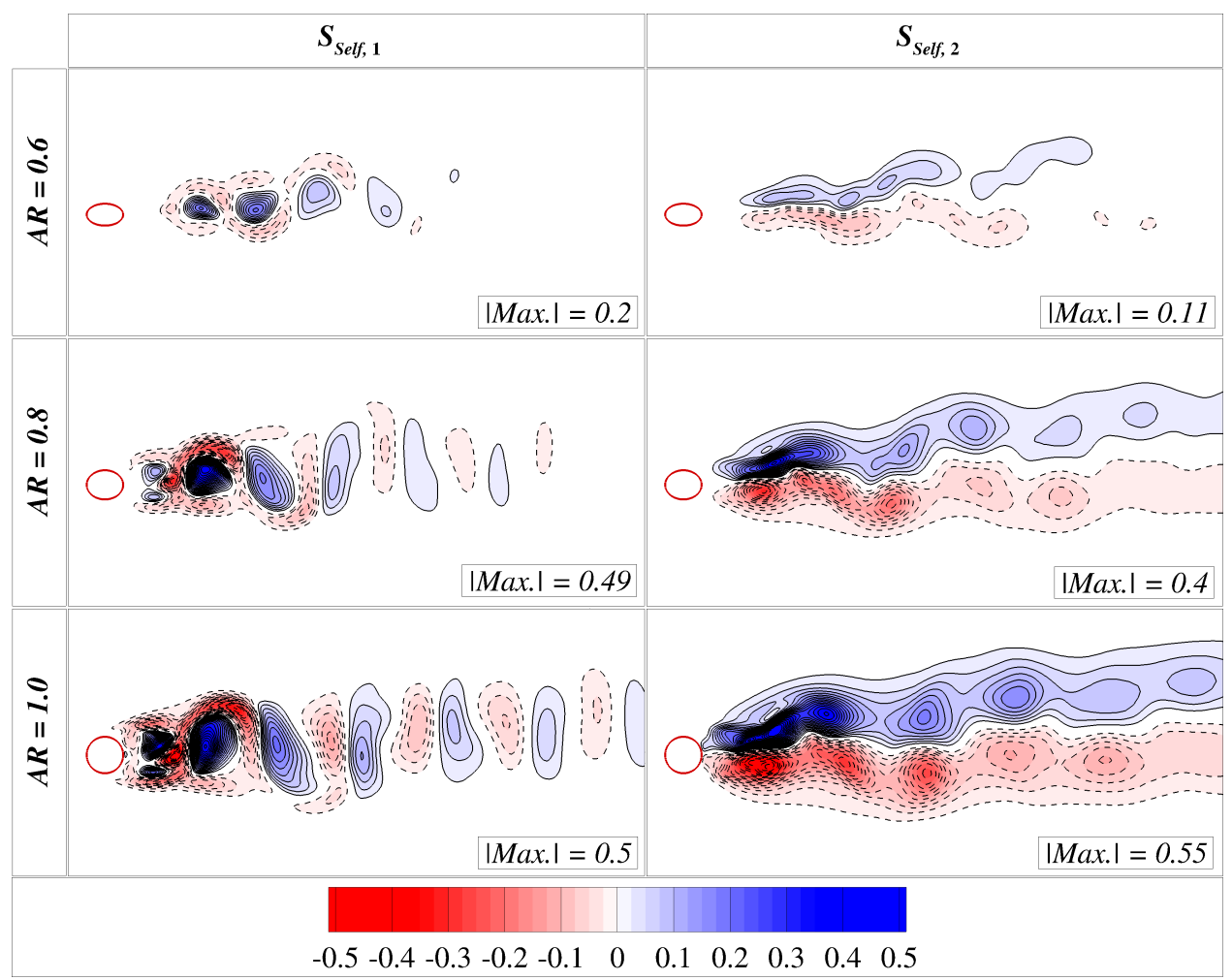

Figure 12: Contours of $S_{\text {self, } 1}$ and $S_{\text {self,2 }}$ terms in Eq. (3.10a) for flow past an elliptic cylinder with different aspect ratios at $R e=130$.

Cheong et al. [1] have explained that Eq. (3.10a) and Eq. (3.10b) denote the selfnoise source term $\left(S_{\mathrm{self}, i}\right)$ due to interaction between velocity fluctuations and shearnoise term $\left(S_{\text {shear }, i}\right)$ arised due to interaction between the mean and the fluctuating velocity components, respectively. Relative importance of the self and the shear-noise source terms in contributing to the aeroacoustic sound generation mechanism was investigated [1]. Cheong et al. [1] found that the contribution of the approximated Lighthill's stress tensor in the mean flow-direction and in the direction perpendicular to the mean flow are comparable and contribution due to shear-noise term is significant over the self-noise term [1] at such high Reynolds numbers. Steady term $S_{0, i}$ denoted by Eq. (3.10c) does not contribute to generation of sound waves as it shows interaction between the mean quantities [1].

Equivalent sound source terms of Eqs. (3.10a) and (3.10b) are evaluated for the different cases investigated in the present work. Fig. 12 shows the variation in intensity of self noise $\left(S_{\text {self }, 1}\right.$ and $\left.S_{\text {self }, 2}\right)$ with aspect ratio at $R e=130$. These self noise terms are evaluated at an instant corresponding to maximum lift coefficient for each aspect ratio case. Strength of the self-noise sources increases with increase in aspect ratio due to increasing bluff body nature. For the case of a circular cylinder $(A R=1)$, 


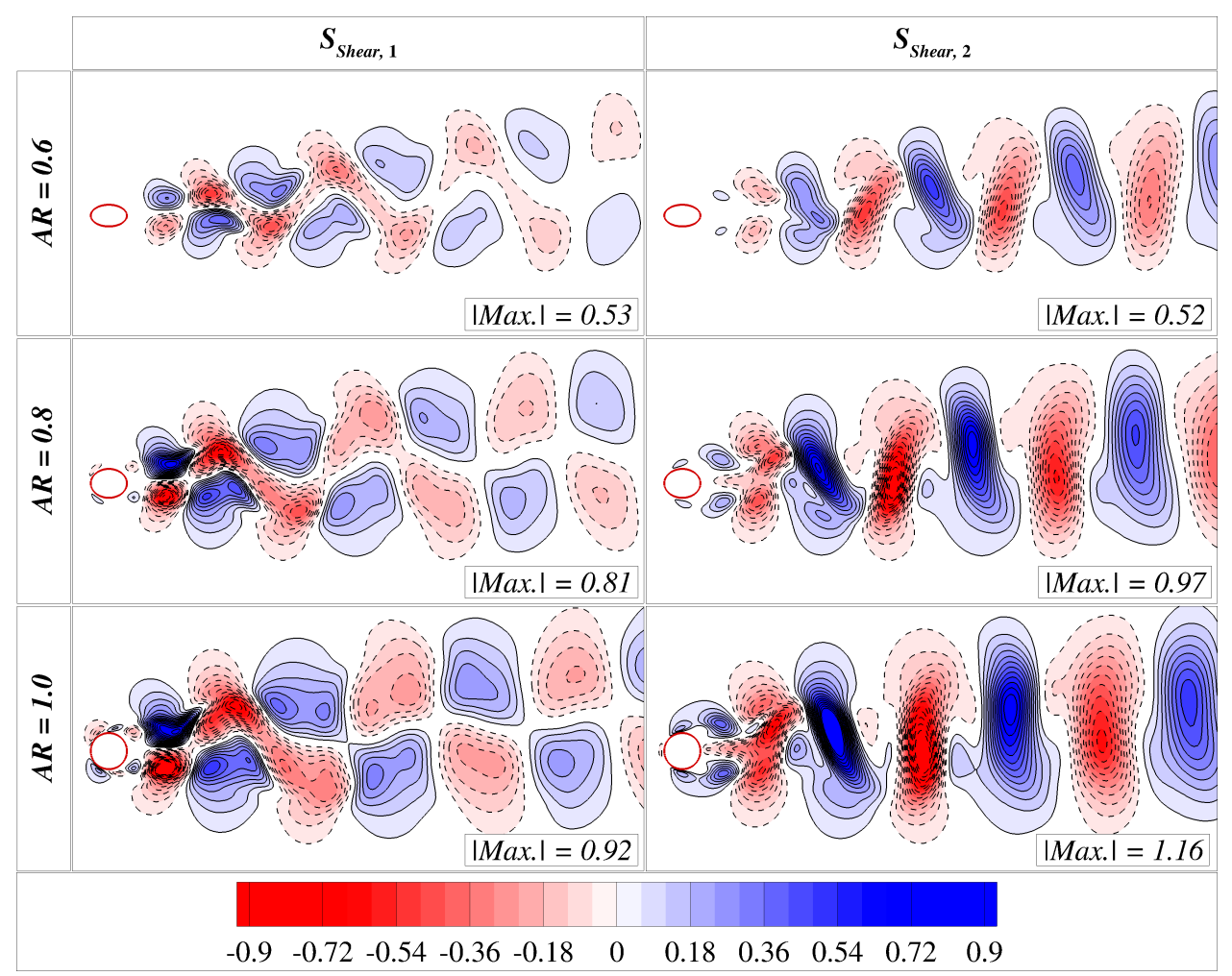

Figure 13: Contours of $S_{\text {shear, }, 1}$ and $S_{\text {shear }, 2}$ terms in Eq. (3.10b) for flow past an elliptic cylinder with different aspect ratios at $R e=130$.

plots show that the $S_{\text {self }, 2}$ term is dominant over the $S_{\text {self, } 1}$ term. However, as body becomes more streamlined for low aspect ratio cases, $S_{\text {self, } 1}$ term is dominant over the $S_{\text {self }, 2}$ term. Arrangement of the equivalent noise sources in the wake region also differs for the self-noise terms. In case of $S_{\text {self }, 1}$ term, noise sources are arranged in a single row with alternative positive and negative structures. The noise sources corresponding to the $S_{\text {self }, 2}$ term are arranged in two parallel rows along the wake region. It is also observed that the $S_{\text {self }, 2}$ term displays structures stretched in the stream-wise direction as compared to $S_{\text {self }, 1}$ term for which structures are elongated normal to the flow direction. One also observes that with increase in the aspect ratio of the elliptic cylinder, noise source terms are formed closer to the body surface.

Variation in intensity of shear-noise sources $\left(S_{\text {shear }, 1} \& S_{\text {shear }, 2}\right)$ with aspect ratio has been displayed in Fig. 13 at $R e=130$. Contours have been shown corresponding to the instant at which the fluctuating lift coefficient is maximum. For different aspect ratios, one observes that the strength of $S_{\text {shear, } 1}$ and $S_{\text {shear, } 2}$ terms are comparable and $S_{\text {shear,2 }}$ term becomes dominant over $S_{\text {shear, } 1}$ term as the aspect ratio increases. It is also observed from Figs. 12 and 13 that the contribution of the shear-noise sources to the sound generation is almost two times higher compared to the self-noise sources 


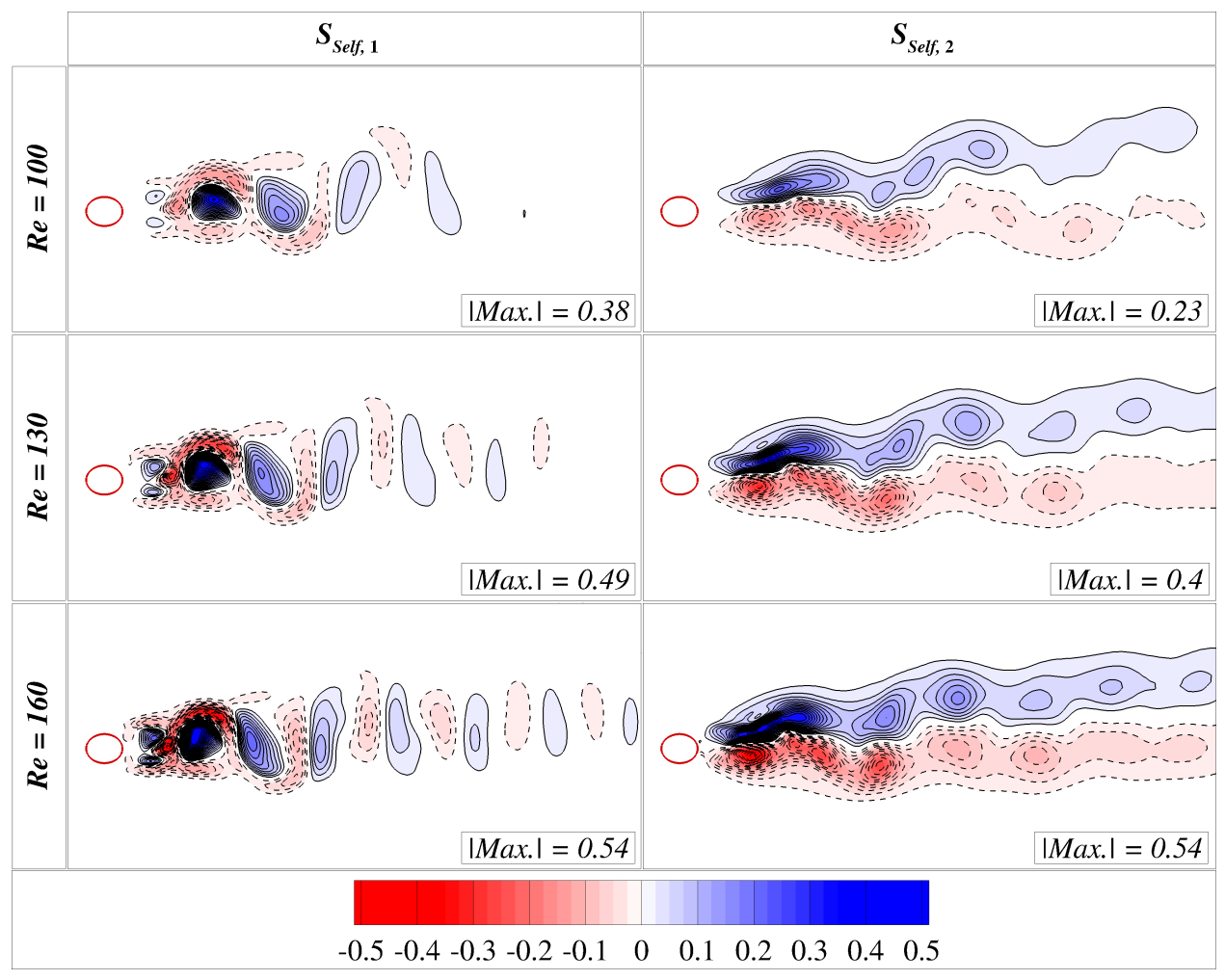

Figure 14: Contours of $S_{\text {self, } 1}$ and $S_{\text {self, } 2}$ terms in Eq. (3.10a) are shown for different Reynolds numbers with $A R=0.8$.

based on the indicated maximum values of sources. In case of shear-noise sources, $S_{\text {shear }, 1}$ term displays structures elongated in the stream-wise direction as compared to $S_{\text {shear }, 2}$ term where the contours display structures elongated perpendicular to the flow direction.

Figs. 14 and 15 show effects of variation of the Reynolds number on the sound self and shear-noise source terms, respectively for an aspect ratio of 0.8 . One observes that the strength of the source terms increases with increase in Reynolds number. This is due to increased amount of fluctuations observed at higher Reynolds number as the viscous effects become less significant compared to the effects associated with convective terms. Strength of the shear-noise term is almost twice higher as compared to self-noise terms.

Information about the amplitudes of the self and shear-noise terms is important as it will have effect on sound power emitted due to the presence of elliptic cylinder in a uniform stream. However the time variation of these source terms dictate the frequency and hence the wavelength of the emitted sound waves. Fig. 16 shows FFT of the time varying self and shear-noise source terms evaluated at a location $(x, y)=(1.0,0.5)$ for indicated values of aspect ratio and Reynolds number. The dominant peak of self-noise $S_{\text {self, } 1}$ term is observed at $2 f_{o}$ which is same as the frequency of corresponding drag co- 


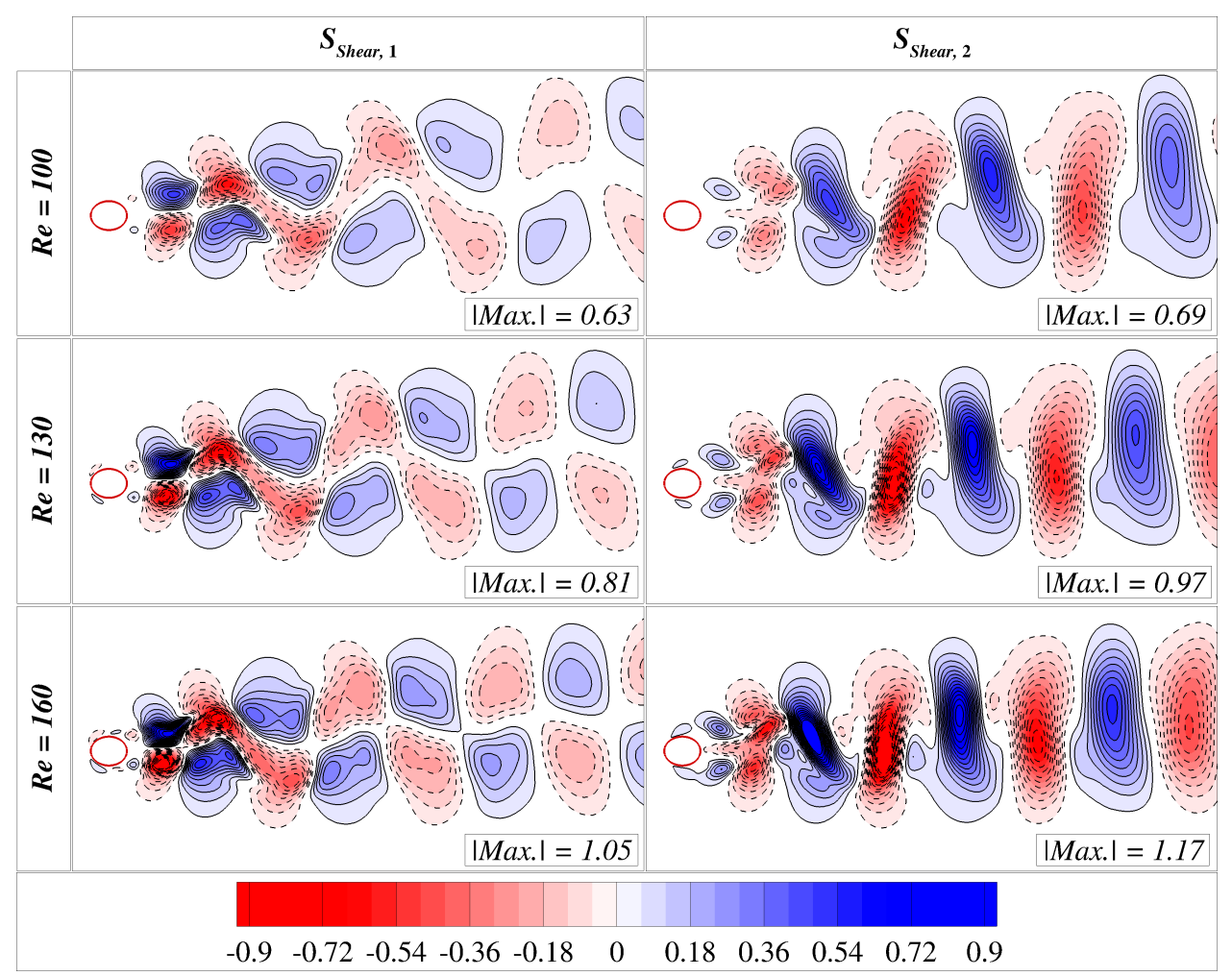

Figure 15: Contours of $S_{\text {shear, } 1}$ and $S_{\text {shear,2 }}$ terms in Eq. (3.10b) are shown for different Reynolds numbers with $A R=0.8$.

efficient while the dominant peaks of $S_{\text {self }, 2}$ are identified at $f_{o}$ and $2 f_{o}$. The shear-noise terms predominantly vary at the frequency corresponding to the lift fluctuations. The contribution of self and shear-noise terms increases with increase in Reynolds number as well as aspect ratio. However, the increase in amplitudes of self noise terms with aspect ratio and Reynolds number are relatively higher than those observed for shear noise terms.

Thus from Figs. 12-16, one concludes that the contribution of the noise source terms increases with increase in the Reynolds number and the aspect ratio. Time variation of the equivalent noise source terms displays the fluctuating lift coefficient frequency and its super-harmonics. The self and the shear-noise terms remain significant only to a small region in the wake, close to the surface of the cylinder.

\subsection{Analysis of disturbance pressure fields}

Next, we have computed the instantaneous pressure fluctuations $p^{\prime}(x, y, t)$ which forms disturbance pressure field using Eq. (3.1). Effects on variation of aspect ratio and Reynolds number on the generated sound have been studied. 

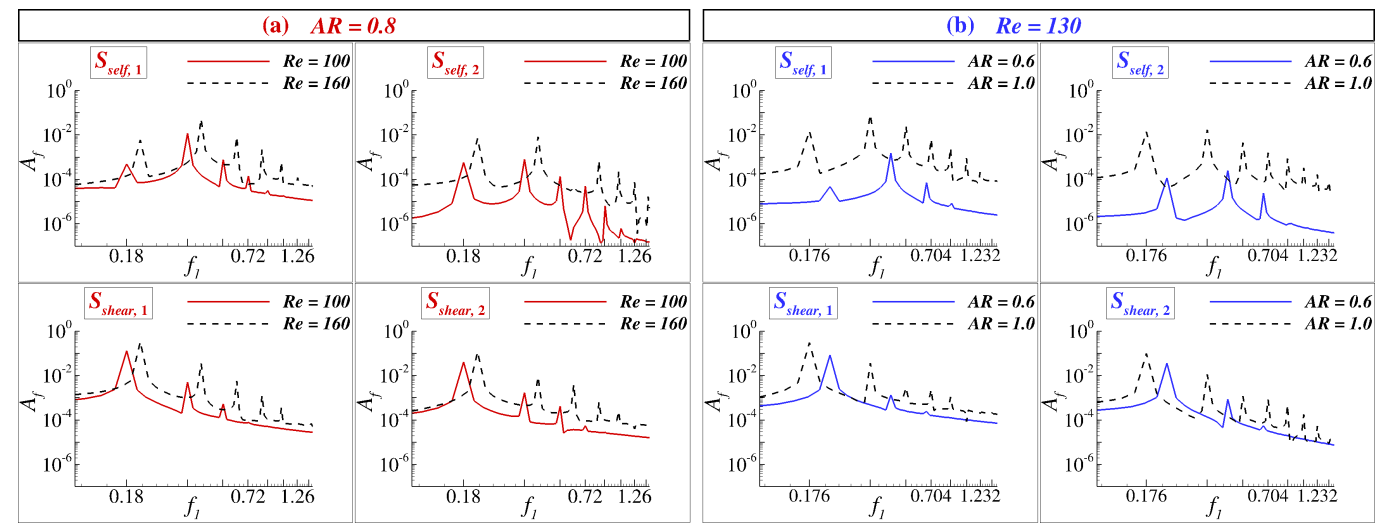

Figure 16: FFTs of time varying self and shear-noise source terms are obtained at a location $(x, y)=$ $(1,0.5)$. Frame (a) represents frequency peaks obtained for $R e=100 \& 160$ with $A R=0.8$. Frame (b) shows frequency peaks obtained for $A R=0.6 \& 1.0$ at $R e=130$.

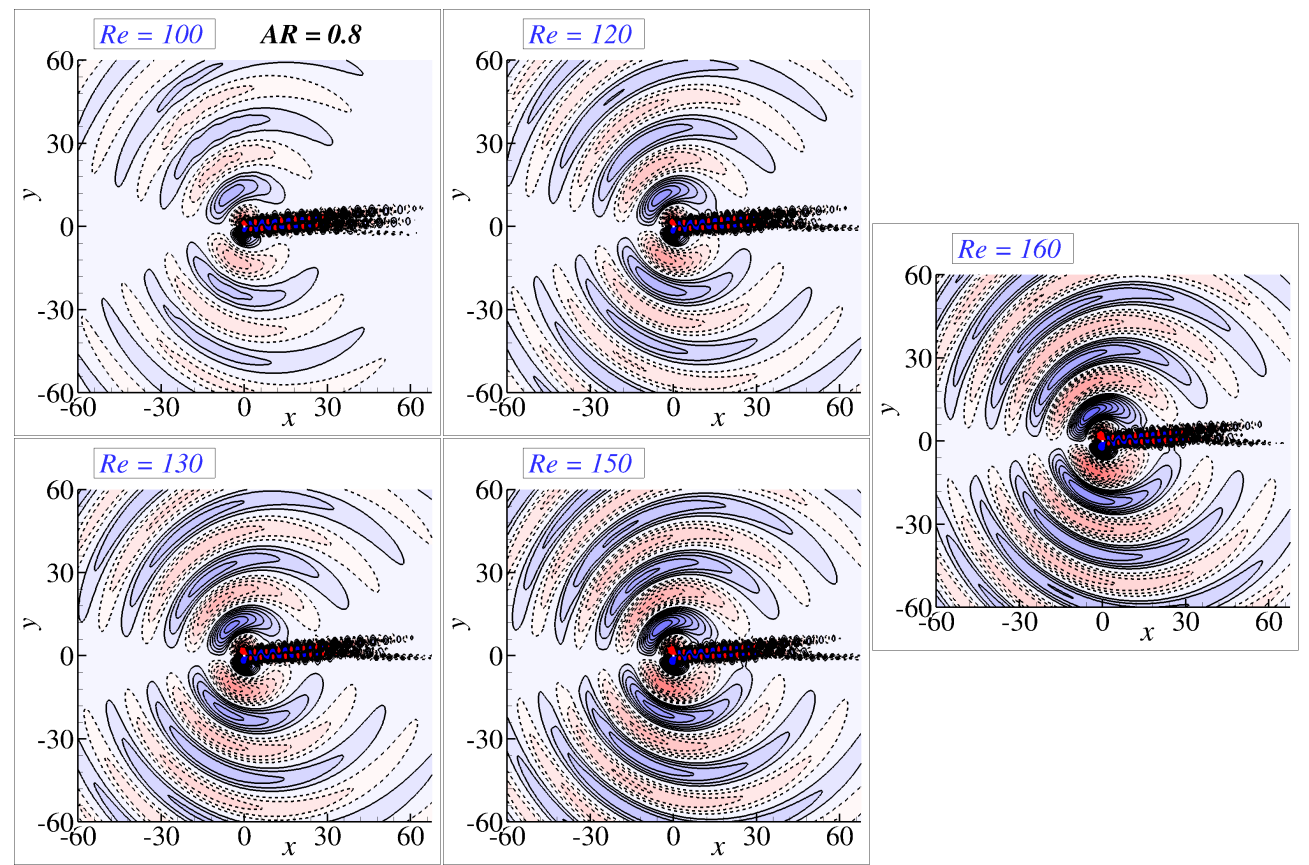

Figure 17: Variation of instantaneous disturbance pressure field $p^{\prime}(x, y, t)$ with Reynolds number at $A R=$ 0.8 . Contour levels vary from -0.008 to 0.008 with 32 equi-spaced intervals. Solid and dashed lines represent positive and negative values of $p^{\prime}$.

Fig. 17 shows the effect of Reynolds number on disturbance pressure fields obtained at $A R=0.8$. Regions of compression and rarefaction are denoted by solid and dashed lines, respectively. It is clearly visible that the intensity of $p^{\prime}(x, y, t)$ has been increased with increase in Reynolds number. This is due to increase in strength of equivalent 


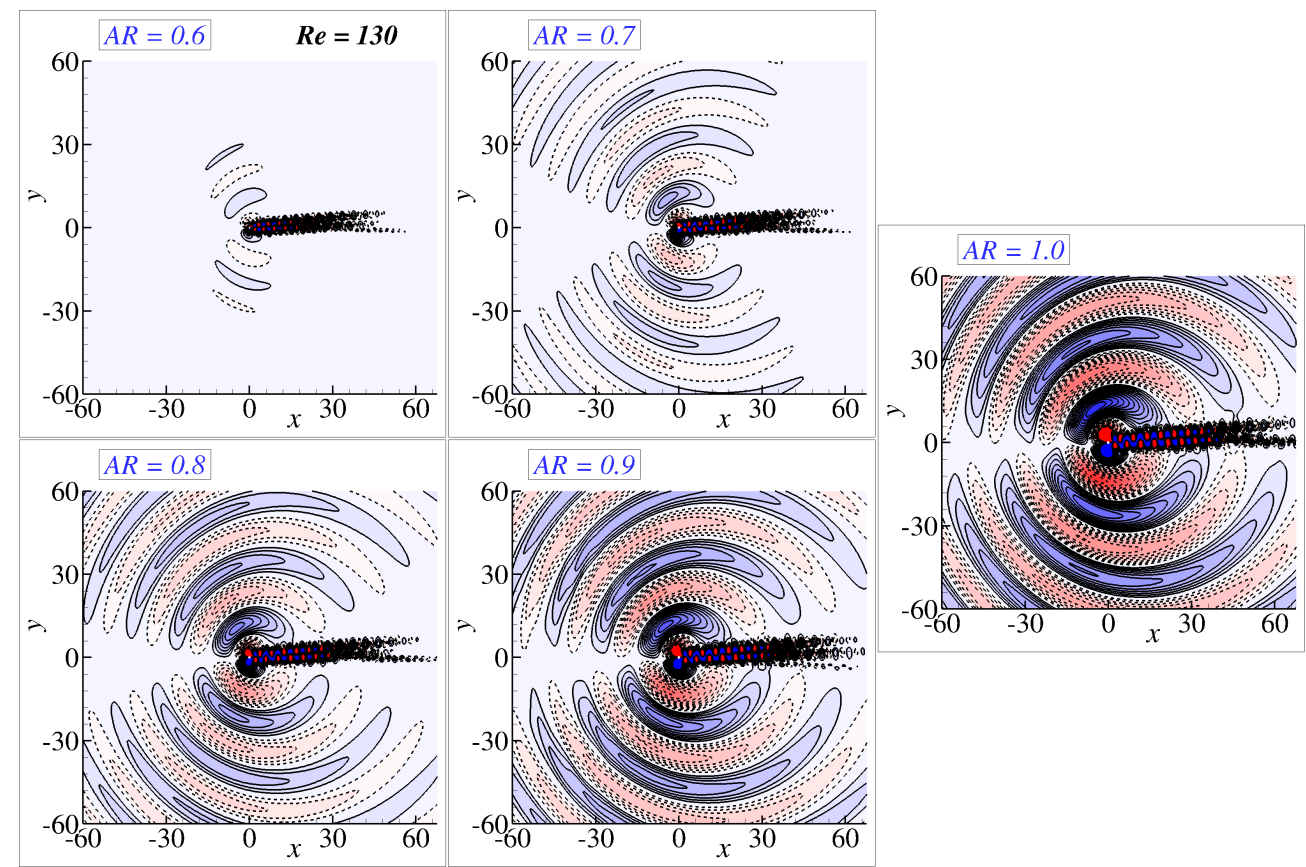

Figure 18: Variation of instantaneous disturbance pressure field $p^{\prime}(x, y, t)$ with aspect ratio at $R e=130$. Contour levels vary from -0.008 to 0.008 with 32 equi-spaced intervals. Solid and dashed lines represent positive and negative values of $p^{\prime}$.

sound noise sources with increase in Reynolds number (as shown in Figs. 7, 14, 15 and 16(a)). Disturbance pressure pulses are found to be propagating almost normal to the direction of free-stream and this results in net radiated sound field dominated by the lift dipole. This has been justified as the amplitudes of lift fluctuations are significantly higher than drag fluctuations for all values of Reynolds number (as displayed in Figs. 9 and 10).

Next, effect of aspect ratio on disturbance pressure fields has been represented in Fig. 18 for $R e=130$. It is observed that intensity of $p^{\prime}(x, y, t)$ increases with increase in aspect ratio, which is expected as the strength of noise sources increases with increase in value of aspect ratio (as shown in Figs. 8, 12, 13 and 16(b)). As the lift fluctuations are significantly dominant over drag fluctuations (as shown in Figs. 9 and 10), the disturbance pressure pulses are observed to propagate along a direction normal to free-stream.

Therefore, disturbance pressure field intensities are analyzed and are related to the strength of equivalent sound noise sources evaluated from acoustic analogies for various values of aspect ratio and Reynolds number. Directivity patterns of radiated sound fields are also obtained using root mean square $R M S$ values of disturbance pressure $\left(p_{R M S}^{\prime}\right)$ for all values of aspect ratio and Reynolds number considered in the present study. 


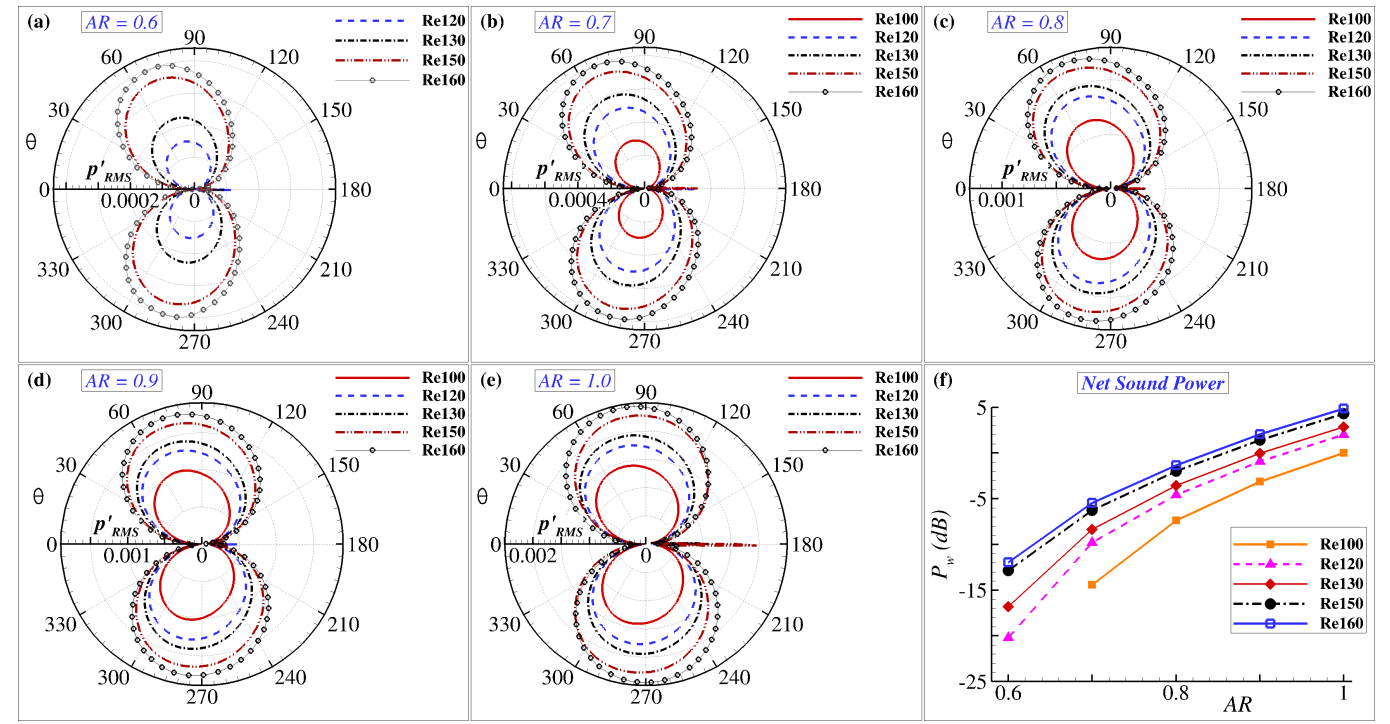

Figure 19: Directivity patterns are obtained using $R M S$ values of $p^{\prime}$ evaluated at a radial distance $r=75$ for all values of aspect ratio and Reynolds number. Frames (a)-(e) represent the variation of $p_{R M S}^{\prime}$ with Reynolds number for indicated values of aspect ratio. Frame (f) shows variation of net sound power $P_{w}$ with aspect ratio for the chosen values of Reynolds number at radius, $r=75$.

Variation of directivity patterns based on $R M S$ values of $p^{\prime}$ with Reynolds number has been shown in Figs. 19(a)-(e) for indicated aspect ratio values. Directivity patterns of generated sound fields are dominated by lift dipole for all values of aspect ratio and Reynolds number. This has been justified as amplitudes of lift fluctuations are much higher as compared to that of drag fluctuations (as shown in Figs. 9 and 10). It is observed that intensity of $p_{R M S}^{\prime}$ increases with increase in Reynolds number for every aspect ratio value. For a given Reynolds number, intensity of $p_{R M S}^{\prime}$ also found to be increasing with aspect ratio. This is due to increase in strength of noise sources with increase in values of Reynolds number and aspect ratio.

In order to find the net sound power output, sound power has been evaluated as [7,21,23],

$$
W=\int_{r=75} I_{a}(r, \theta) r d \theta
$$

where, $I_{a}=\frac{p_{R M S}^{\prime}}{\rho_{\infty} U_{\infty}}$ accounts for mean sound intensity in the far-field region. The net sound power level in decibels $(d B)$ is evaluated as $[7,21,23]$,

$$
P_{w}=20 \log _{10} \frac{W}{W_{o}},
$$

where, $W_{o}$ corresponds to the reference sound power obtained for flow past a circular cylinder $A R=1.0$ for $R e=100$. 


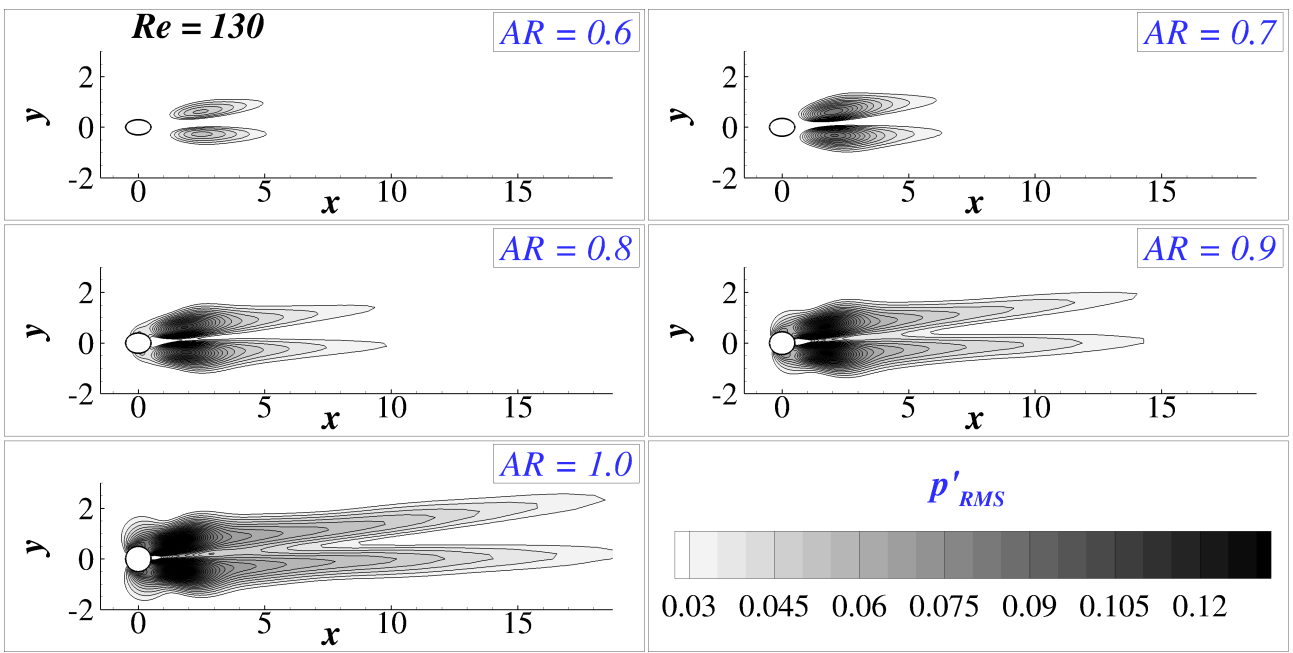

Figure 20: Overall pressure fluctuations in the wake region are estimated using the $R M S$ values of disturbance pressure field $p_{R M S}^{\prime}$ for different values of aspect ratio at $R e=130$. Minimum and maximum values of $p_{R M S}^{\prime}$ are considered as 0.03 and 0.13 with 21 intervals, respectively.

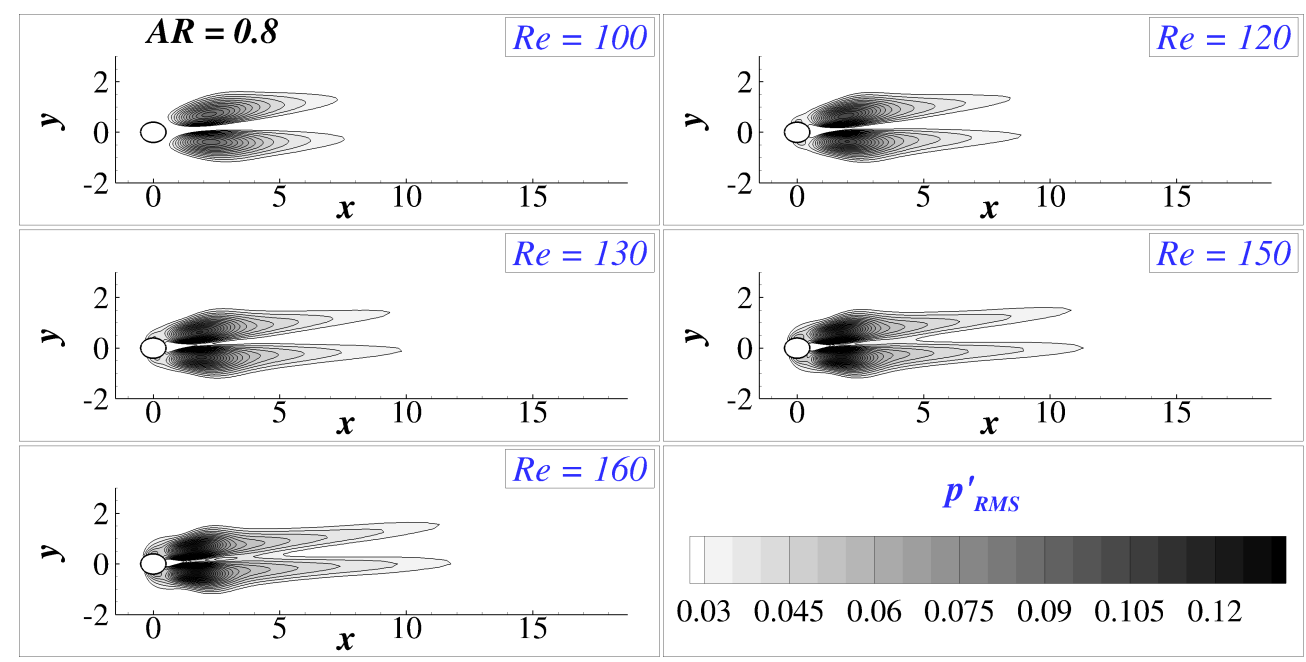

Figure 21: Overall pressure fluctuations in the wake region are estimated using the $R M S$ values of disturbance pressure field $p_{R M S}^{\prime}$ for different values of Reynolds number at $A R=0.8$. Minimum and maximum values of $p_{R M S}^{\prime}$ are considered as 0.03 and 0.13 with 21 intervals, respectively.

Fig. 19(f) represents variation of net radiated sound power $P_{w}$ with aspect ratio for different value of Reynolds number. Net sound power displays an increasing trend with increase of Reynolds number and aspect ratio. Minimum and maximum values of $P_{w}$ are observed for $R e=120 \& A R=0.6$ and $R e=160 \& A R=1.0$, respectively. Trends of $P_{w}$ with variation of aspect ratio and Reynolds number are exactly similar to the trends observed for equivalent noise sources associated with acoustic analogies (as 
shown in Figs. 7 to 16). Therefore, the generated disturbance pressure fields, directivity patterns and net radiated sound power due to flow past elliptic cylinder at different Reynolds numbers are analyzed and are related to intensity of equivalent noise sources obtained from acoustic analogies.

Overall intensity of pressure fluctuations in the wake region are estimated by evaluating the $R M S$ values of disturbance pressure fields $p_{R M S}^{\prime}$. Fig. 20 show the contours of $p_{R M S}^{\prime}$ in the wake region for indicated values of aspect ratio at $R e=130$. The contour levels vary from 0.03 to 0.13 with 21 equi-spaced intervals. The intensity of $p_{R M S}^{\prime}$ in the wake region increases with increase in aspect ratio and there by increasing the overall strength of generated sound field at higher values of aspect ratio (shown in Fig. 19(f)).

Fig. 21 displays the contours of $p_{R M S}^{\prime}$ in the wake region for different Reynolds numbers at $A R=0.8$. It is observed there is an increase in overall pressure fluctuations in the wake with increase in Reynolds number. Due to this reason the overall strength of generated sound field has been increased with increase in Reynolds number (shown in Fig. 19(f)).

\subsection{Proper orthogonal decomposition of disturbance pressure fields}

In the literature, proper orthogonal decomposition $(P O D)$ technique has been adopted to understand and analyze complex fluid dynamic problems by obtaining different significant coherent structures $[12,33,34,37]$. In the present study, $P O D$ analysis has been implemented for time varying disturbance pressure fields in order to identify and quantify several significant $P O D$ modes. $P O D$ analysis has been implemented using method of snapshots [37]. $P O D$ analysis for disturbance pressure fields has been carried out in order to identify $P O D$ modes corresponding to the lift and the drag dipoles of radiated sound fields $[7,21]$. Instantaneous disturbance pressure field is expressed as a linear superposition of spatial $P O D$ modes $\left(\phi_{k}(x, y)\right)$ along with time-varying $P O D$ amplitudes $\left(a_{n}(t)\right)$ as given by [33],

$$
p^{\prime}(x, y, t)=\sum_{k=1}^{N} a_{k}(t) \phi_{k}(x, y),
$$

where, $N$ accounts for number of snapshots. $\phi_{k}(x, y)$ and $\lambda_{k}$ are eigenfunctions or eigenmodes and eigenvalues of a covariance matrix $R_{i j}$ [33]. The value of $\lambda_{k}$ corresponding to $\phi_{k}(x, y)$ signifies the amount of contribution by $k^{\text {th }}$ mode to the disturbance pressure field. The sum of all the normalized values of $\lambda_{k}$ is unity $\left(\sum_{k=1}^{n} \lambda_{k}=1\right)$. Further details of $P O D$ implementation have been adopted from the available literature $[21,33]$. The $P O D$ analysis uses orthogonal basis functions $\phi_{k}(x, y)$. The phase difference between any two different $P O D$ modes is known to be $90^{\circ}$ [33].

Time varying disturbance pressure fields are considered for more than 15 lift cycles. Here, $P O D$ analysis has been applied for flow past two different elliptic cylinders with $A R=0.6$ and $A R=1.0$ for $R e=130$. 

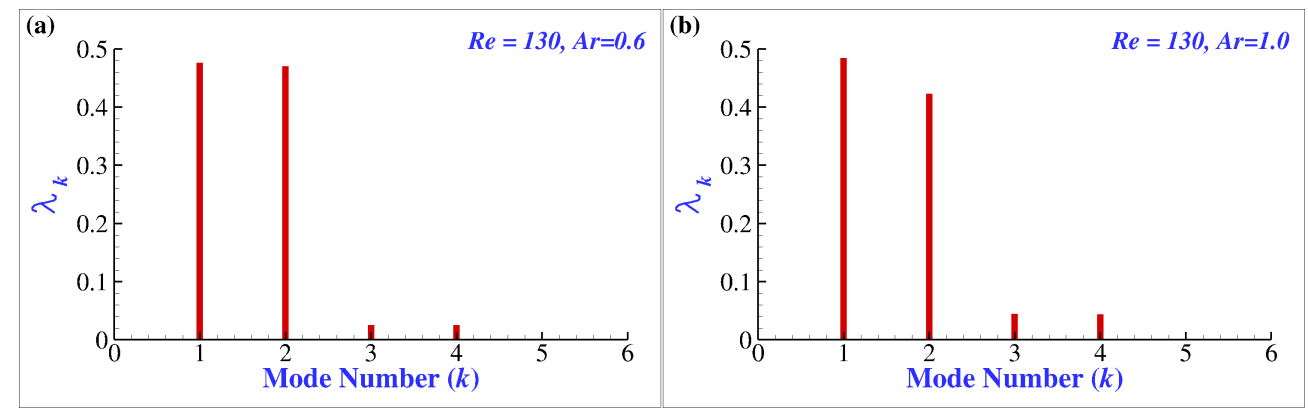

Figure 22: The variation of the modal energy associated with each $P O D$ mode has been expressed in terms of $\lambda_{k}$ values for $A r=0.6 \& 1.0$ cases at $R e=130$. The first four $P O D$ modes are significant enough to contribute more than $99 \%\left(\lambda_{1}+\lambda_{2}+\lambda_{3}+\lambda_{4}>0.99\right)$ to the disturbance pressure field.

The contribution of individual $P O D$ modes $\left(\lambda_{k}\right)$ to the disturbance pressure field has been obtained for $A R=0.6 \& 1.0$ cases at $R e=130$. Fig. 22 shows the variation of $\lambda_{k}$ against $k^{t h}$ mode for $A R=0.6 \& 1.0$ cases. It is clear that the first four modes (with mode numbers $k=1,2,3 \& 4$ ) are sufficient enough to contribute more than $99 \%\left(\lambda_{1}+\lambda_{2}+\lambda_{3}+\lambda_{4}>0.99\right)$ to the disturbance pressure field with more than $90 \%$ contribution $\left(\lambda_{1}+\lambda_{2}>0.90\right)$ from the first two modes $(k=1 \& 2)$ for both values of aspect ratio.

Fig. 23 represents first four dominant $P O D$ modes $\phi_{k}(x, y)$ and their time varying $P O D$ amplitudes $a_{n}(t)$ which are obtained for $A R=0.6$ with $R e=130$. These first four $P O D$ modes contribute more than $99 \%$ to the disturbance pressure field based on the values of $\lambda_{k}$. Solid and dashed lines denote positive and negative values of $P O D$ modes, respectively. Contour levels vary from minimum (Min) to maximum (Max) with 30 equi-spaced levels. The first two modes (Mode 1 and Mode 2) form a pair showing similar coherent structures with almost equal contribution $\left(\lambda_{1} \approx \lambda_{2}\right)$ to the disturbance pressure field with a phase difference of $90^{\circ}$ between $a_{1}(t)$ and $a_{2}(t)$. Similarly, the Mode 3 and Mode 4 with almost equal energy content $\left(\lambda_{3} \approx \lambda_{4}\right)$ form a second mode pair with a phase difference of $90^{\circ}$ between $a_{3}(t)$ and $a_{4}(t)$. Several studies in the past has mentioned the occurrence of mode pair when the coherent structures of two different eigenmodes resemble with each other and their eigenvalues are almost equal [33]. Disturbance pressure field can also be represented in terms of instability modes as [5,33],

$$
p^{\prime}(x, y, t)=\sum_{j=1}^{\infty}\left[A_{j}(t) f_{j}(x, y)+A_{j}^{*}(t) f_{j}^{*}(x, y)\right],
$$

where, $f_{j}$ and $f_{j}^{*}$ space-dependent modes occurring in pair. The terms with asterisk denote the complex conjugates. In Fig. 23, disturbance pulses in the first mode pair (Mode 1 and Mode 2) are found to propagate along the normal direction of free-stream (resembles to a lift dipole), while the next two modes (Mode 3 and Mode 4) propagate 
(a)

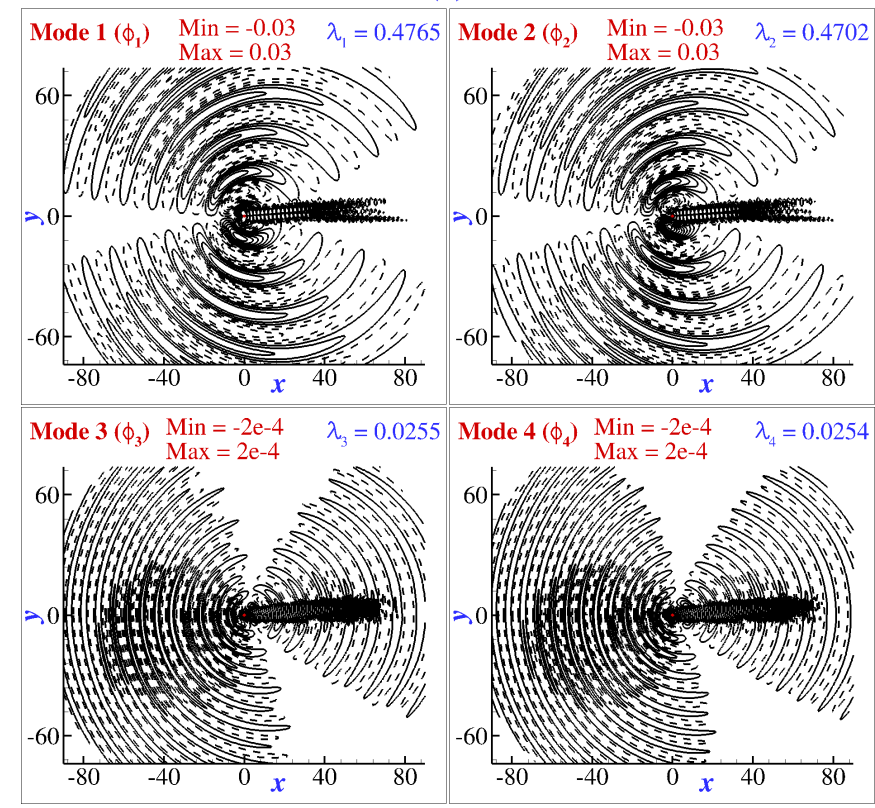

(b)

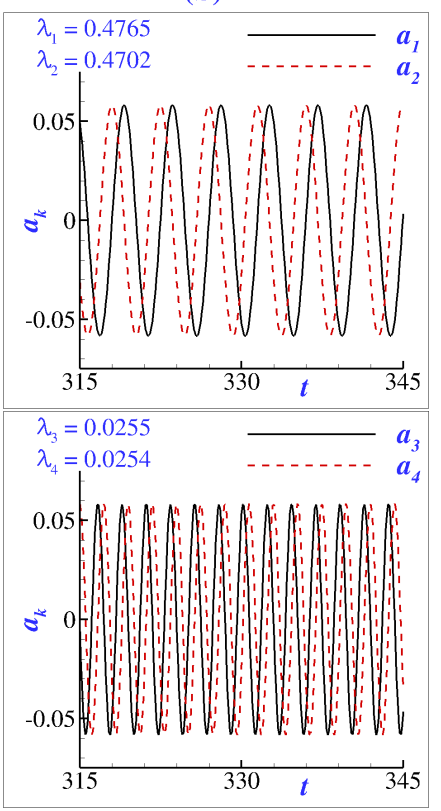

Figure 23: (a) First four dominant $P O D$ modes $\phi_{k}(x, y)$ of disturbance pressure field are shown for $A R=0.6$ with $R e=130$. Here, Modes $1 \& 2$ contribute to lift dipole whereas Modes $3 \& 4$ contribute to drag dipole. (b) Time varying $P O D$ amplitudes $a_{k}$ are shown here. Frequencies of $a_{1} \& a_{2}$ and $a_{3} \& a_{4}$ are observed as shedding frequency $f_{o}$ and twice the shedding frequency $2 f_{o}$, respectively.

along the free-stream direction (resembles to a drag dipole). Spatial frequency of second mode pair (Mode 3 and Mode 4) is almost twice that the frequency observed for first mode pair (Mode 1 and Mode 2). Frequencies of $a_{1} \& a_{2}$ and $a_{3} \& a_{4}$ are found to be corresponding to the vortex shedding frequency $f_{o}$ and twice the shedding frequency $2 f_{o}$, respectively. It is known that the alternate shedding of vortices has resulted in the fluctuations of lift and drag forces acting on the cylinder, and the frequencies of lift and drag forces are known to be shedding frequency $f_{o}$ (same as for $a_{1}(t)$ and $a_{2}(t)$ ) and twice the shedding frequency $2 f_{o}$ (same as for $a_{3}(t)$ and $a_{4}(t)$ ), respectively. According to Curle's acoustic analogy, the fluctuations in lift and drag forces are responsible for the lift and the drag dipoles, respectively. Therefore, the first two modes (Mode 1 and Mode 2) contributing more than $94 \%$ to the disturbance pressure fields are associated with lift dipole, whereas the next two modes (Mode 3 and Mode 4) contributing less than $6 \%$ are associated with the drag dipole. Hence, the obtained POD modes have confirmed that the disturbance pressure fields are significantly dominated by the lift dipole.

First four dominant $P O D$ modes and respective $P O D$ coefficients of $p^{\prime}(x, y, t)$ obtained for $A R=1.0$ with $R e=130$ are displayed in Fig. 24. First four $P O D$ modes contribute more than $99 \%$ to the disturbance pressure field. Frequencies of $a_{1}$ and $a_{2}$ are same and equal to the shedding frequency $f_{o}$, whereas frequencies of $a_{3}$ and $a_{4}$ 
(a)

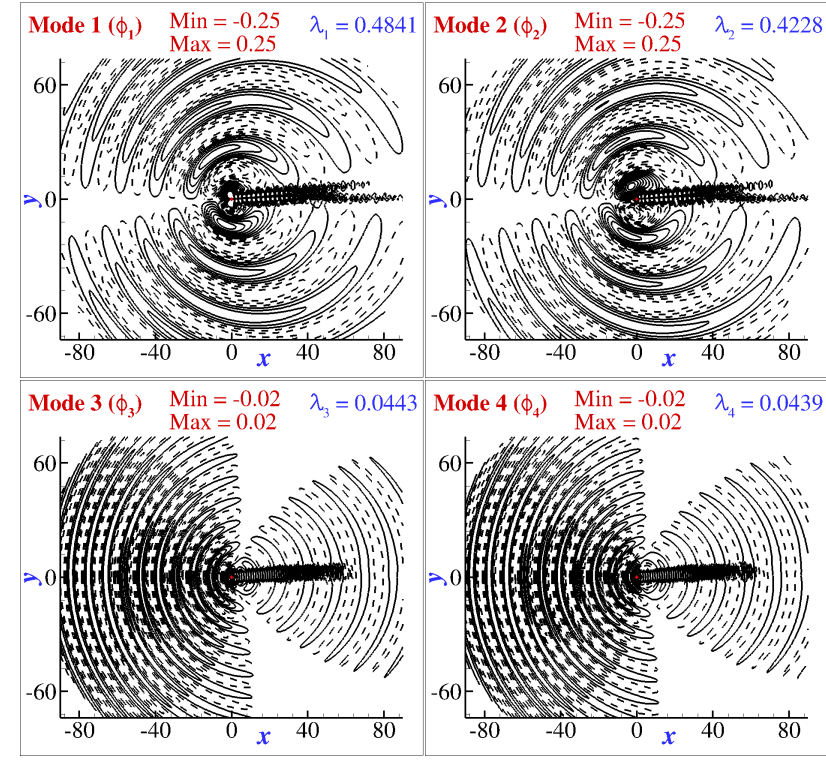

(b)

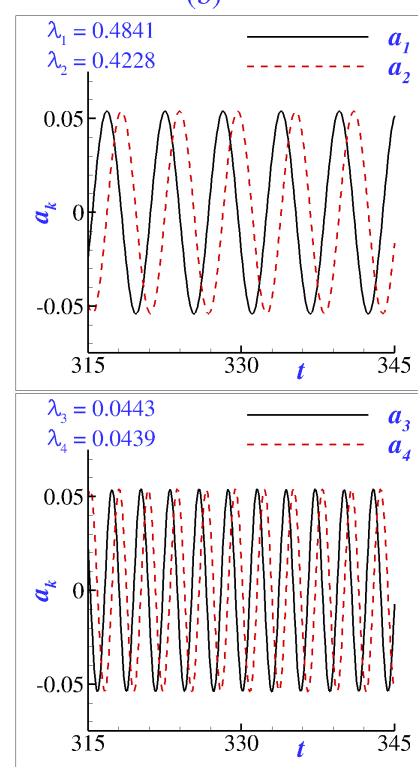

Figure 24: (a) First four dominant $P O D$ modes $\phi_{k}(x, y)$ of disturbance pressure field are shown for $A R=1.0$ with $R e=130$. Here, Modes $1 \& 2$ contribute to lift dipole whereas Modes 3 \& 4 contribute to drag dipole. (b) Time varying $P O D$ amplitudes $a_{k}$ are shown here. Frequencies of $a_{1} \& a_{2}$ and $a_{3} \& a_{4}$ are observed as the shedding frequency $f_{o}$ and twice the shedding frequency $2 f_{o}$, respectively.

are found to be twice the shedding frequency $2 f_{o}$. Similar to $A R=0.6$ case, first two $P O D$ modes of $A R=1.0$ case contributing around $91 \%$ to $p^{\prime}(x, y, t)$ are related to the lift dipole and the next two $P O D$ modes contributing more than $8 \%$ are related to the drag dipole. Therefore, the disturbance pressure fields are found to be dominated by the lift dipole.

Thus, $P O D$ analysis of disturbance pressure fields has helped us to identify and quantify $P O D$ modes related to lift and drag dipoles. It has also confirmed that disturbance pressure fields are dominated by the lift dipole based on the values of $\lambda_{k}$.

\subsection{Doak's decomposition}

Doak has decomposed momentum density field $(\rho \mathbf{u})$ into acoustic, hydrodynamic and thermal modes as given by [4],

$$
\rho \mathbf{u}=\overline{\mathbf{B}}+\mathbf{B}^{\prime}-\nabla \psi^{\prime}
$$

where, $\bar{B}$ and $B^{\prime}$ account for the mean and the fluctuating solenoidal components, respectively such that $\nabla \cdot \overline{\mathbf{B}}=0$ and $\nabla \cdot \mathbf{B}^{\prime}=0$. Hydrodynamic fluctuations of momentum density field are denoted by fluctuating solenoidal component $\mathbf{B}^{\prime}$. Here, fluctuating scalar potential function $\psi^{\prime}$ accounts for irrotational field and for time stationary flows, mean component of irrotational field is assumed to be zero. Irrotational 


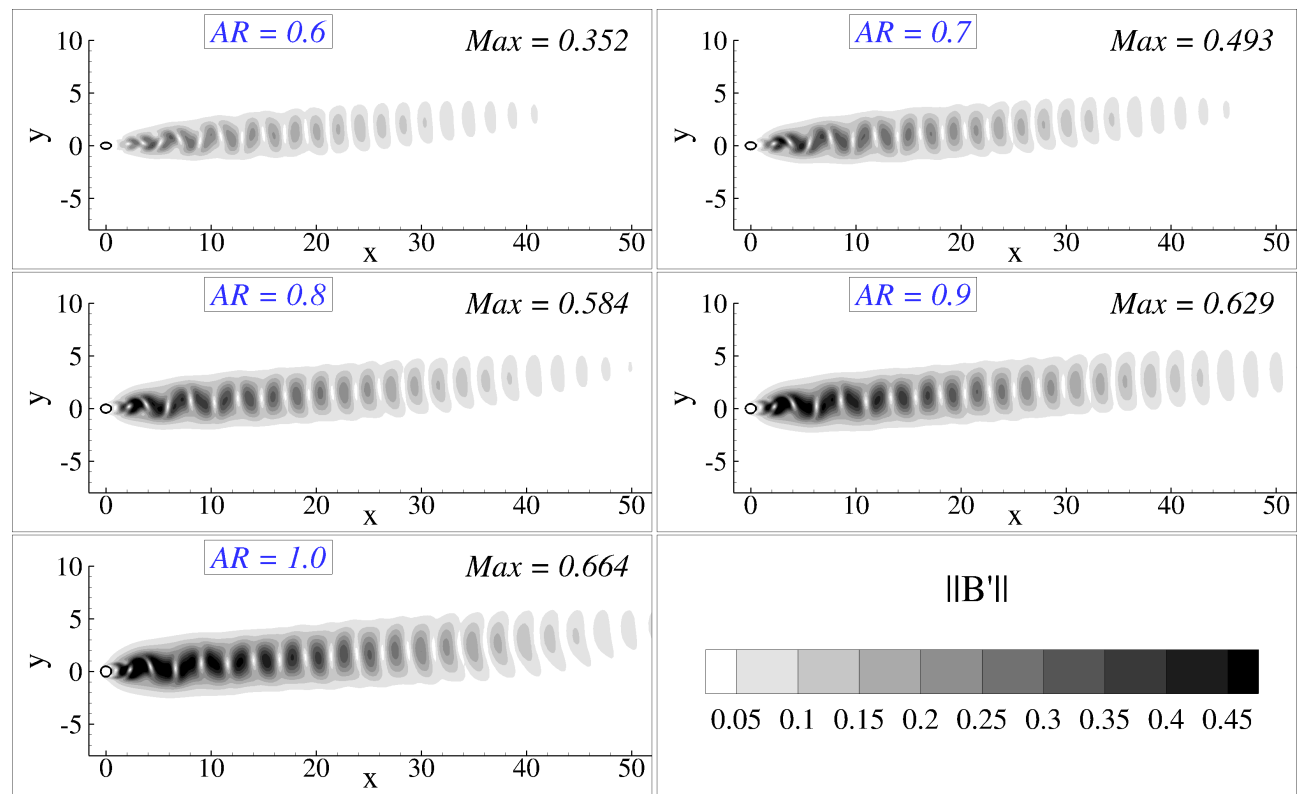

Figure 25: Using Doak's decomposition technique, variation in intensity of hydrodynamic modes $\left\|\mathbf{B}^{\prime}\right\|$ with aspect ratio for $R e=130$ has been shown.

component $\psi^{\prime}$ is further decomposed into acoustic and thermal modes given as $[4,41]$,

$$
\psi^{\prime}=\psi_{T}^{\prime}+\psi_{A}^{\prime}
$$

Fluctuating scalar potential function $\psi^{\prime}$ and acoustic component $\psi_{A}^{\prime}$ are evaluated using following equations $[4,41]$,

$$
\begin{aligned}
\nabla^{2} \psi^{\prime} & =\frac{\partial \rho^{\prime}}{\partial t}, \\
\nabla^{2} \psi_{A}^{\prime} & =\frac{1}{c_{\infty}^{2}} \frac{\partial p^{\prime}}{\partial t} .
\end{aligned}
$$

Numerical procedure for solving these Eqs. (3.15), (3.16) and (3.17)) to obtain acoustic $\left\|\nabla \psi_{A}^{\prime}\right\|$ and hydrodynamic $\left\|\mathbf{B}^{\prime}\right\|$ modes has been explained by Mahato et al. [21] and is not reported here to avoid repetition. In the present study, Doak's decomposition has been implemented for different values of aspect ratio at $R e=130$ to study the effect of aspect ratio on the intensity of hydrodynamic and acoustic modes.

Fig. 25 represents variation in intensity of hydrodynamic fluctuations $\left\|\mathbf{B}^{\prime}\right\|$ with aspect ratio at $R e=130$. It has been observed that the hydrodynamic fluctuations are mostly concentrated in the wake region. The strength of hydrodynamic fluctuations has been increased with increase in aspect ratio value with minimum and maximum intensities are observed at $A R=0.6$ and $A R=1.0$, respectively. Acoustic modes $\left\|\nabla \psi_{A}^{\prime}\right\|$ obtained for different values of aspect ratio at $R e=130$ are shown 


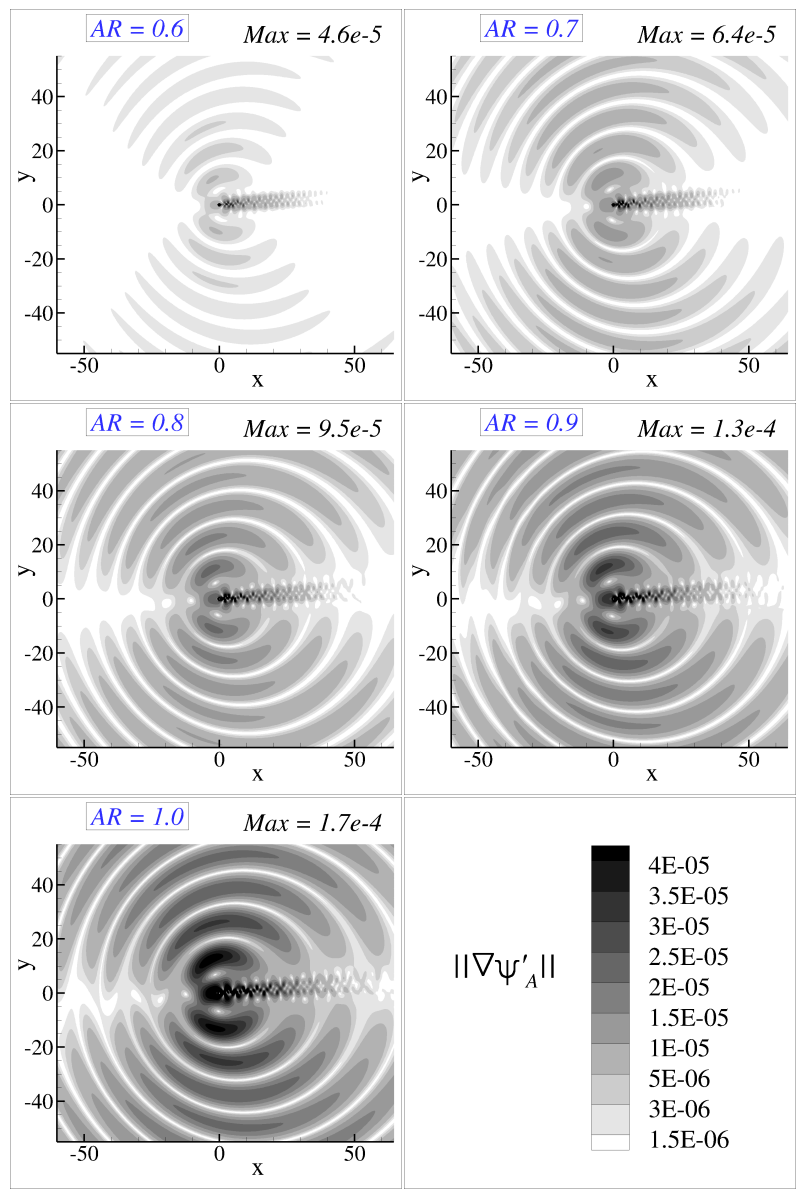

Figure 26: Using Doak's decomposition technique, variation in intensity of acoustic modes $\left\|\nabla \psi_{A}^{\prime}\right\|$ with aspect ratio for $R e=130$ has been shown.

in Fig. 26. These acoustic modes are propagating along the direction normal to freestream and are found to be exactly similar to the disturbance pressure fields as shown in Fig. 18. As aspect ratio value increases from 0.6 to 1.0, strength of acoustic modes has increased gradually with minimum and maximum intensities obtained at $A R=0.6$ and $A R=1.0$, respectively. This is due to increase in intensity of hydrodynamic fluctuations with aspect ratio value. Therefore, Doak's decomposition technique confirms that the radiated sound (sound power $P_{w}$ ) increases with increase in aspect ratio (as shown in Fig. 19(f)).

\section{Summary and conclusions}

In the present analysis, direct simulations have been performed to analyze the sound fields generated due to unsteady laminar flow over elliptic cylinders for a Reynolds 
number range $100 \leq R e \leq 160$. Simulations have been performed for a free-stream Mach number $M=0.2$ with $4^{\circ}$ angle of attack. Aspect ratio of elliptic cylinder is considered in the range $0.6 \leq A R \leq 1.0$. Unsteady two dimensional compressible fluid flow equations are solved in the conservative form using high resolution spatiotemporal $O C C D-O R K 5$ schemes. Results show that both flow field and sound field characteristics are sufficiently resolved over long radial distances. Intensity of sound fields has been significantly affected by variation of aspect ratio and Reynolds number. Following are the important conclusions obtained from the present simulations.

1. Information about equivalent sound noise source terms have been extracted with the help of Powell's acoustic analogy, Curle's acoustic analogy, approximated Lighthill's stress tensor and the Doak's decomposition technique.

2. The source term associated with the Powell's analogy and the solenoidal fluctuations given by the Doak's decomposition technique provide information about the hydrodynamic fluctuations present in the wake region which are responsible for sound generation.

3. Approximated Lighthill's stress tensor provides information about the self-noise and shear-noise terms highlighting the interaction between either velocity fluctuations alone or the interaction between the mean and fluctuating velocity components. Contribution of the shear-noise source terms to sound generation is significantly higher as compared to the self-noise source terms and their time variation corresponds to the variation of lift coefficient.

4. Equivalent noise sources evaluated using acoustic analogies become progressively intense with increase in Reynolds number as well as aspect ratio. These source patterns are related to the corresponding disturbance pressure field intensities.

5. Directivity patterns based on $R M S$ values of $p^{\prime}$ show dipolar nature dominated by the lift dipole. Net sound power intensity has been increased with increase in Reynolds number and aspect ratio. Minimum and maximum values of net radiated sound power are found at $R e=120 \& A R=0.6$ and $R e=160 \&$ $A R=1.0$, respectively.

6. Amplitudes of time varying lift and drag fluctuations are increased with Reynolds number and aspect ratio. However, amplitudes of lift fluctuations are significantly greater than the amplitudes observed for drag fluctuations. Hence, it is justified that the resultant sound fields exhibit dipolar behavior dominated by the lift dipole.

7. Using the $P O D$ analysis of disturbance pressure fields, $P O D$ modes associated with the lift and the drag dipoles have been identified and quantified for the chosen values of aspect ratio and Reynolds number. Also, $P O D$ analysis has confirmed that the resultant sound fields are dominated by lift dipole based on the values of $\lambda_{k}$. 
8. Hydrodynamic and acoustic modes are separated using Doak's decomposition technique. Variation in strength of acoustic modes with aspect ratio has shown similar behavior when compared to the disturbance pressure fields shown in Fig. 18.

Thus the present $D N S$ approach successfully computed sound radiated due to flow past elliptic cylinders at various Reynolds numbers. Present $D N S$ approach also provides useful information about the equivalent noise sources which correlated well with the obtained sound power for various cases considered in the present work.

\section{References}

[1] C. Cheong, P. Joseph, Y. PARK And S. Lee, Computation of aeolian tone from a circular cylinder using source models, Appl. Acous., 69(2) (2008), pp. 110-126.

[2] N. CuRLE, The influence of solid boundaries upon aerodynamic sound, Proc. R. Soc. Lond. A, 231 (1955), pp. 505-514.

[3] A. Dipankar AND T. Sengupta, Symmetrized compact scheme for receptivity study of $2 D$ transitional channel flow, J. Comput. Phys., 215(1) (2006), pp. 245-273.

[4] P. DOAK, Momentum potential theory of energy flux carried by momentum fluctuations, J. Sound Vib., 131(1) (1989), pp. 67-90.

[5] P. G. Drazin AND W. H. ReID, Hydrodynamic Stability, Cambridge University Press, 2004.

[6] B. Etkin, G. K. Korbacher And R. T. Keefe, Acoustic radiation from a stationary cylinder in a finite stream (aeolian tones), J. Acoust. Soc. Am., 29 (1957), pp. 30-36.

[7] N. GantA, B. Mahato AND Y. G. BhumkaR, Analysis of sound generation by flow past a circular cylinder performing rotary oscillations using direct simulation approach, Phys. Fluids, 31(2) (2019), 026104.

[8] J. H. GeRrard, Measurements of the sound from circular cylinders in an air stream, Proc. Phys. Soc. Lond. B, 68 (1955), pp. 453-461.

[9] J. C. HARDin AND D. S. Pope, An acoustic/viscous splitting technique for computational aeroacoustics, Theoret. Comput. Fluid Dyn., 6 (1994), pp. 323-340.

[10] Y. HATTORI AND R. KomATSU, Mechanism of aeroacoustic sound generation and reduction in a flow past oscillating and fixed cylinders, J. Fluid Mech., 832 (2017), pp. 241-268.

[11] K. A. Hoffmann And S. T. Chiang, Computational Fluid Dynamics Volume II, 2000.

[12] P. Holmes, Turbulence, Coherent Structures, Dynamical Systems and Symmetry, Cambridge University Press, 2012.

[13] M. Howe, Contributions to the theory of aerodynamic sound, with application to excess jet noise and the theory of the flute, J. Fluid Mech., 71(4) (1975), pp. 625-673.

[14] O. InOuE, Propagation of sound generated by weak shock-vortex interaction, Phys. Fluids, 12 (2000), pp. 1258-1261.

[15] O. INOUE, Effect of initial condition on the sound generation by flow past a rotary-oscillating circular cylinder, Phys. Fluids, 18(11) (2006), 118106.

[16] O. InOue AND N. HATAKEYAMA, Sound generation by a two-dimensional circular cylinder in a uniform flow, J. Fluid Mech., 471 (2002), pp. 285-314.

[17] O. INOUE AND Y. TAKAHASHI, Successive generation of sounds by shock-strong vortex interaction, Phys. Fluids, 12 (2000), pp. 3229-3234.

[18] S. K. LELE, Compact finite difference schemes with spectral-like resolution, J. Comput. Phys., 103(1) (1992), pp. 16-42. 
[19] M. J. Lighthill, On sound generated aerodynamically: I. General Theory, Proc. R. Soc. Lond. A, 221 (1952), pp. 564-587.

[20] Y. S. K. Liow, B. T. TAN, M. C. Thompson AND K. Hourigan, Sound generated in laminar flow past a two-dimensional rectangular cylinder, J. Sound Vib., 295 (2006), pp. 407-427.

[21] B. Mahato, N. GANTA AND Y. G. Bhumkar, Direct simulation of sound generation by a two-dimensional flow past a wedge, Phys. Fluids, 30(9) (2018), 096101.

[22] B. MAhato, N. GANTA AND Y. G. BhUMKAR, Computation of aeroacoustics and fluid flow problems using a novel dispersion relation preserving scheme, J. Theor. Comput. Acoustics, 26(4) (2019), 1850063.

[23] F. MAR GNAT, Hybrid prediction of the aerodynamic noise radiated by a rectangular cylinder at incidence, Comput. Fluids, 109 (2015), pp. 13-26.

[24] R. Mittal AND S. Balachandar, Direct numerical simulation of flow past elliptic cylinders, J. Comput. Phys., 124 (1996), pp. 351-367.

[25] W. MöHRING, On vortex sound at low mach number, J. Fluid Mech., 85(4) (1978), pp. 685-691.

[26] O. M. Phillips, The intensity of aeolian tones, J. Fluid Mech., 1 (1956), pp. 607-624.

[27] A. Powell, Theory of vortex sound, J. Acoust. Society America, 36 (1964), pp. 177-195.

[28] J. Pradhan, S. Jindal, B. Mahato AND Y. G. BHumkaR, Joint optimization of the spatial and the temporal discretization scheme for accurate computation of acoustic problems, Commun. Comput. Phys., 24(2) (2018), pp. 408-434.

[29] L. RAYleigh, The Theory of Sound, Vols. I \& II, Macmillan, 1896.

[30] S. R. L. Samion, M. S. M. Ali, A. Abu, C. J. Doolan and R. Z. Y. Porteous, Aerodynamic sound from a square cylinder with a downstream wedge, Aero. Sci. Tech., 53 (2016), pp. 85-94.

[31] T. K. SEngupta, High Accuracy Computing Methods: Fluid Flows and Wave Phenomena, Cambridge University Press, USA, 2013.

[32] T. K. SEnGUPTA, S. De AND S. SARKAR, Vortex-induced instability of an incompressible wall-bounded shear layer, J. Fluid Mech., 493 (2003), pp. 277--286.

[33] T. K. Sengupta, N. Singh And V. Suman, Dynamical system approach to instability of flow past a circular cylinder, J. Fluid Mech., 656 (2010), pp. 82-115.

[34] T. K. SENGUPTA, V. VIJAY AND N. SingH, Universal instability modes in internal and external flows, Comput. Fluids, 40(1) (2011), pp. 221-235.

[35] W. Z. SHEN AND J. N. Sorensen, Comment on the aeroacoustic formulation of Hardin and Pope, AIAA J., 37 (1999), pp. 141-143.

[36] K. Shintani, A. Umemura AND A. TAKANo, Low-Reynolds-number flow past an elliptic cylinder, J. Fluid Mech., 136 (1983), pp. 277-289.

[37] L. SiROVICH, Turbulence and the dynamics of coherent structures, part I-III, Q. Appl. Math., 45 (1987).

[38] S. SOUMYA AND K. A. PRAKASH, Effect of splitter plate on passive control and drag reduction for fluid flow past an elliptic cylinder, Ocean Eng., 141 (2017), pp. 351-374.

[39] V. Strouhal, Ueber eine besondere art der tonerregung, Annu. Phys. Chem. Wied. Annu. Phys., 5 (1878), pp. 216-251.

[40] C. K. W. TAM AND J. C. HARDIn, Proc. Second Computational Aeroacoustics (CAA) Workshop on Benchmark Problems, NASA cp-3352.

[41] S. Unnikrishnan AND D. V. Gaitonde, Acoustic, hydrodynamic and thermal modes in a supersonic cold jet, J. Fluid Mech., 800 (2016), pp. 387-432.

[42] M. WANG, S. K. LELE AND P. MoIn, Computation of quadrupole noise using acoustic anal- 
ogy, AIAA J., 34 (1996), pp. 2247-2254.

[43] J. E. F. Williams, Hydrodynamic noise, Annu. Rev. Fluid Mech., 1 (1969), pp. 197-222.

[44] J. E. F. Williams, Aeroacoustics, Annu. Rev. Fluid Mech., 9 (1977), pp. 447-468.

[45] J. E. F. Williams, Aeroacoustics, J. Sound Vib., 190 (1996), pp. 387-398.

[46] C. H. K. Williamson, Oblique and parallel modes of vortex shedding in the wake of a circular cylinder at low Reynolds numbers, J. Fluid Mech., 206 (1989), pp. 579-627.

[47] C. H. K. Williamson, Vortex dynamics in the cylinder wake, Annu. Rev. Fluid Mech., 28 (1996), pp. 477-539. 